\section{UNCONSTRAINED OPTIMIZATION}

\section{Poul Erik Frandsen, Kristian Jonasson \\ Hans Bruun Nielsen, Ole Tingleff}

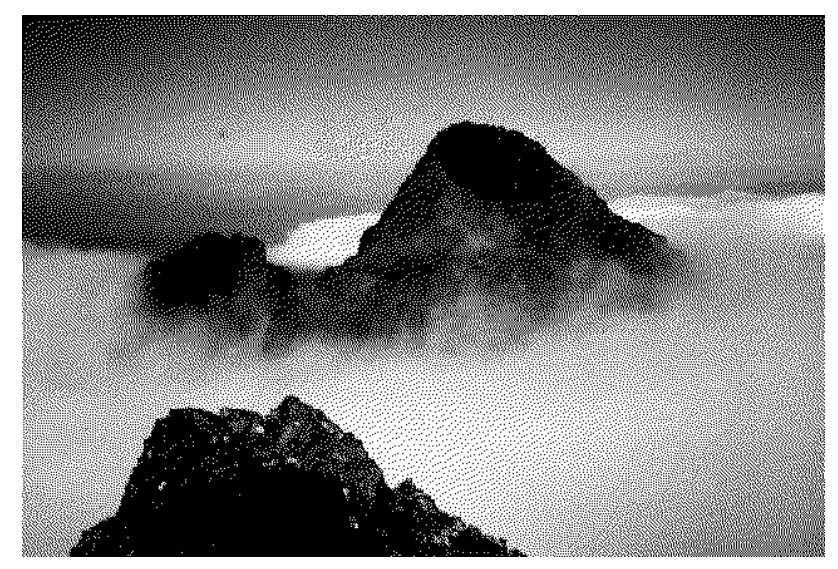

LECTURE NOTE

IMM-LEC-2

\section{ABSTRACT}

This lecture note is intended for use in the course 04212 Optimization and Data Fitting at the Technincal University of Denmark. It covers about $25 \%$ of the curriculum. Hopefully, the note may be useful also to interested persons not participating in that course.

The aim of the note is to give an introduction to algorithms for unconstrained optimization. We present Conjugate Gradient, Damped Newton and Quasi Newton methods together with the relevant theoretical background.

The reader is assumed to be familiar with algorithms for solving linear and nonlinear system of equations, at a level corresponding to an introductory course in numerical analysis.

The algorithms presented in the note appear in any good program library, and implementations can be found via GAMS (Guide to Available Mathematical Software) at the Internet address

http://gams.nist.gov

The examples in the note were computed in Matlab. The programs are available via

http://www.imm.dtu.dk/ hbn/software.html

\title{
$\overline{\mathrm{IMM}}$
}



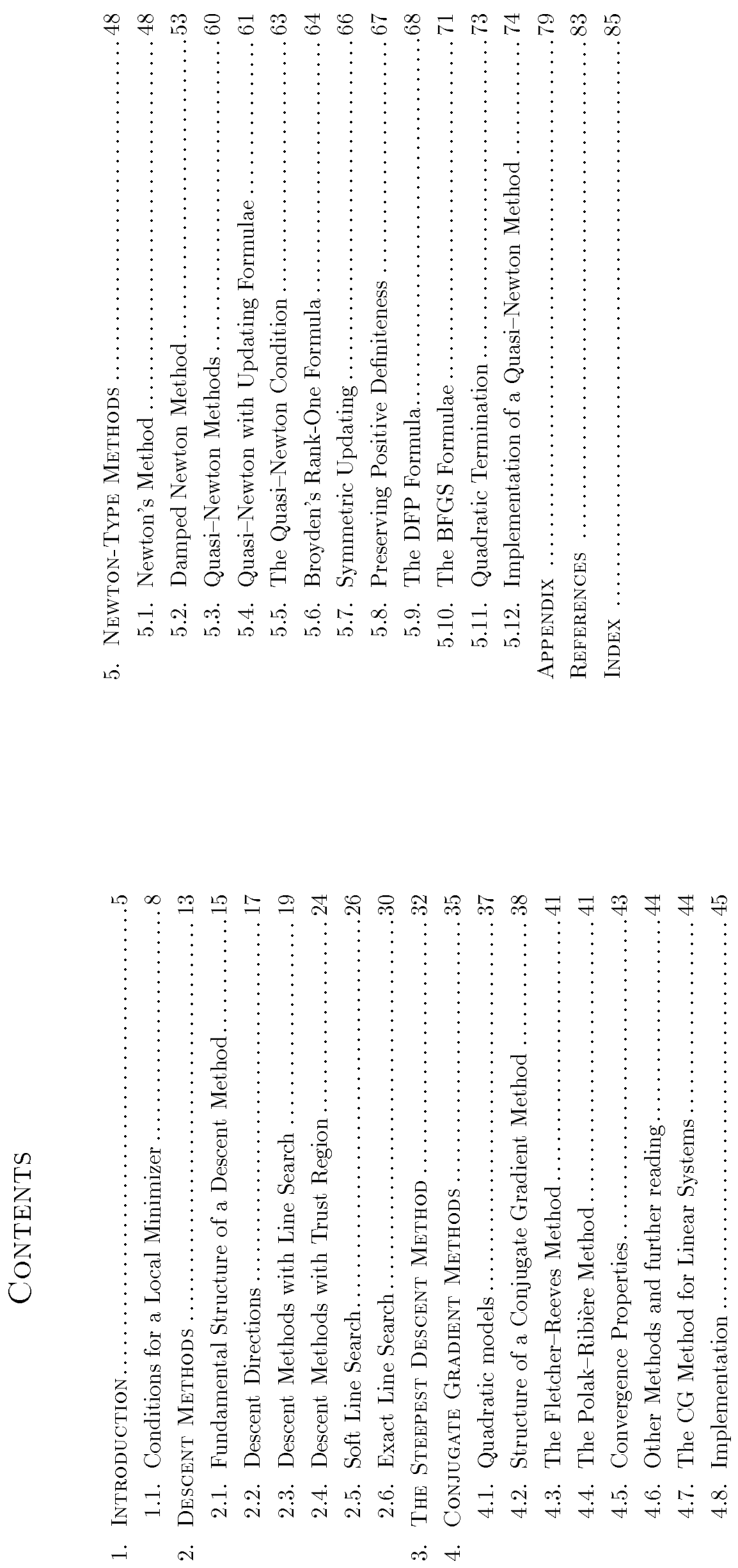

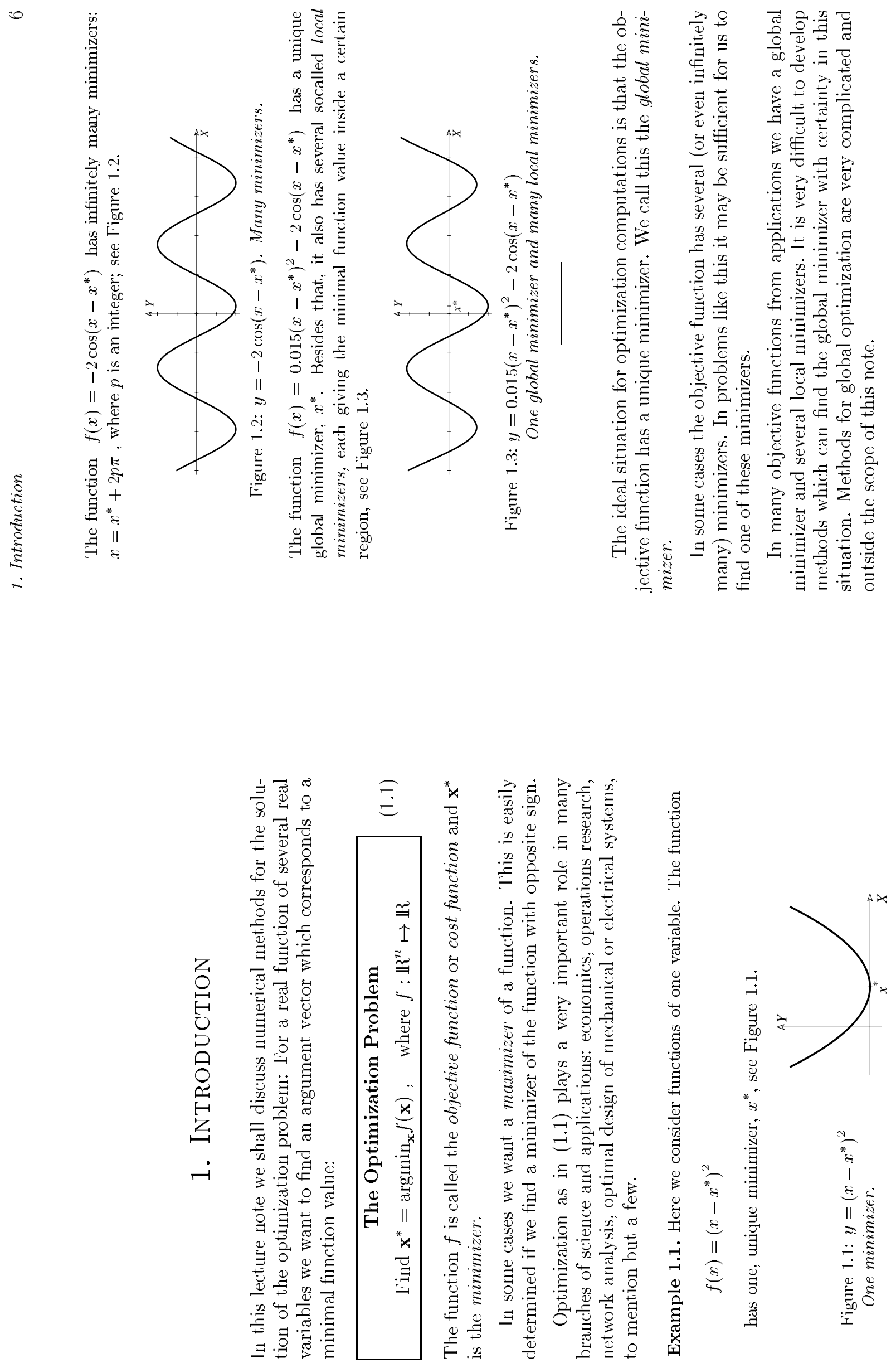

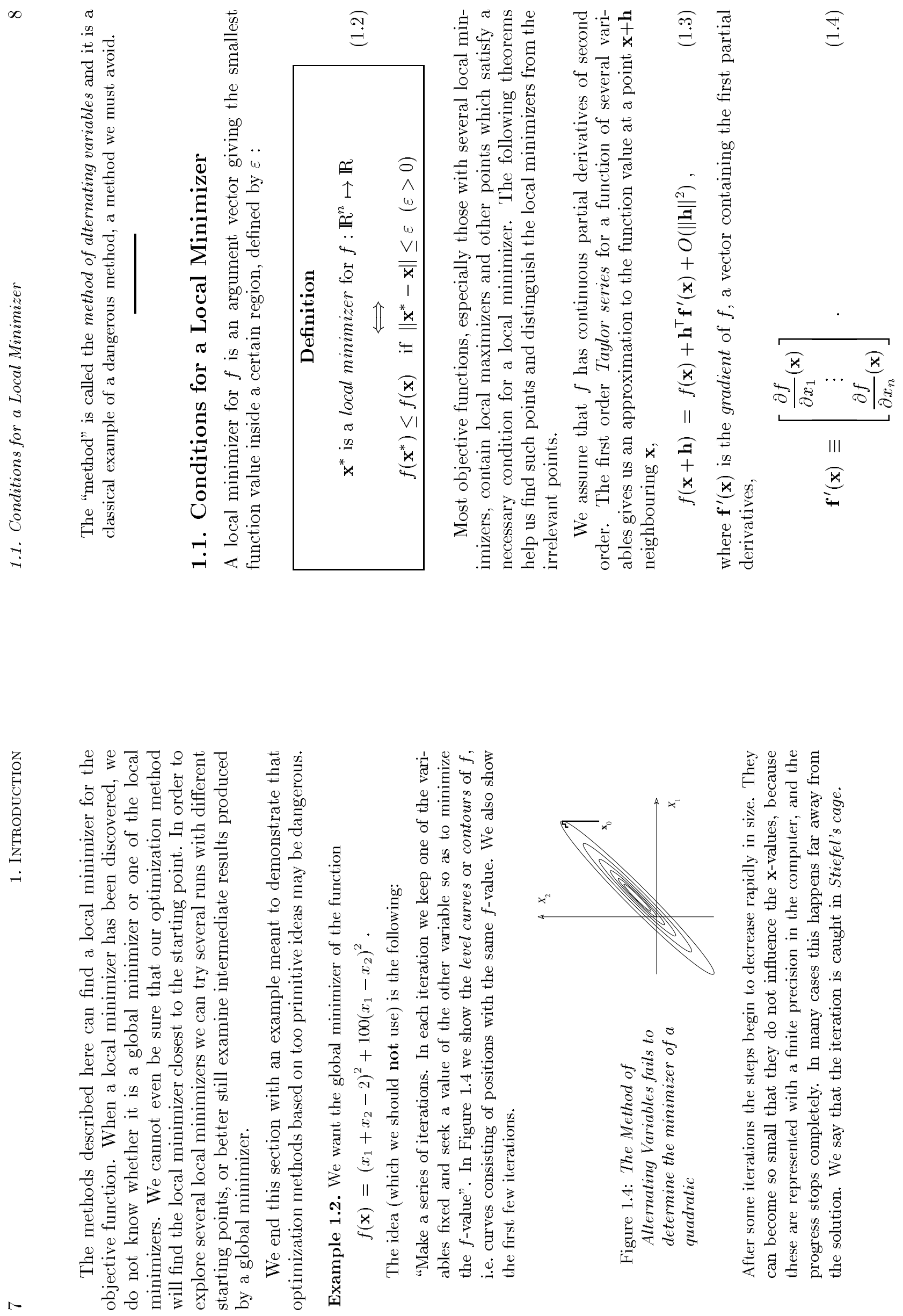

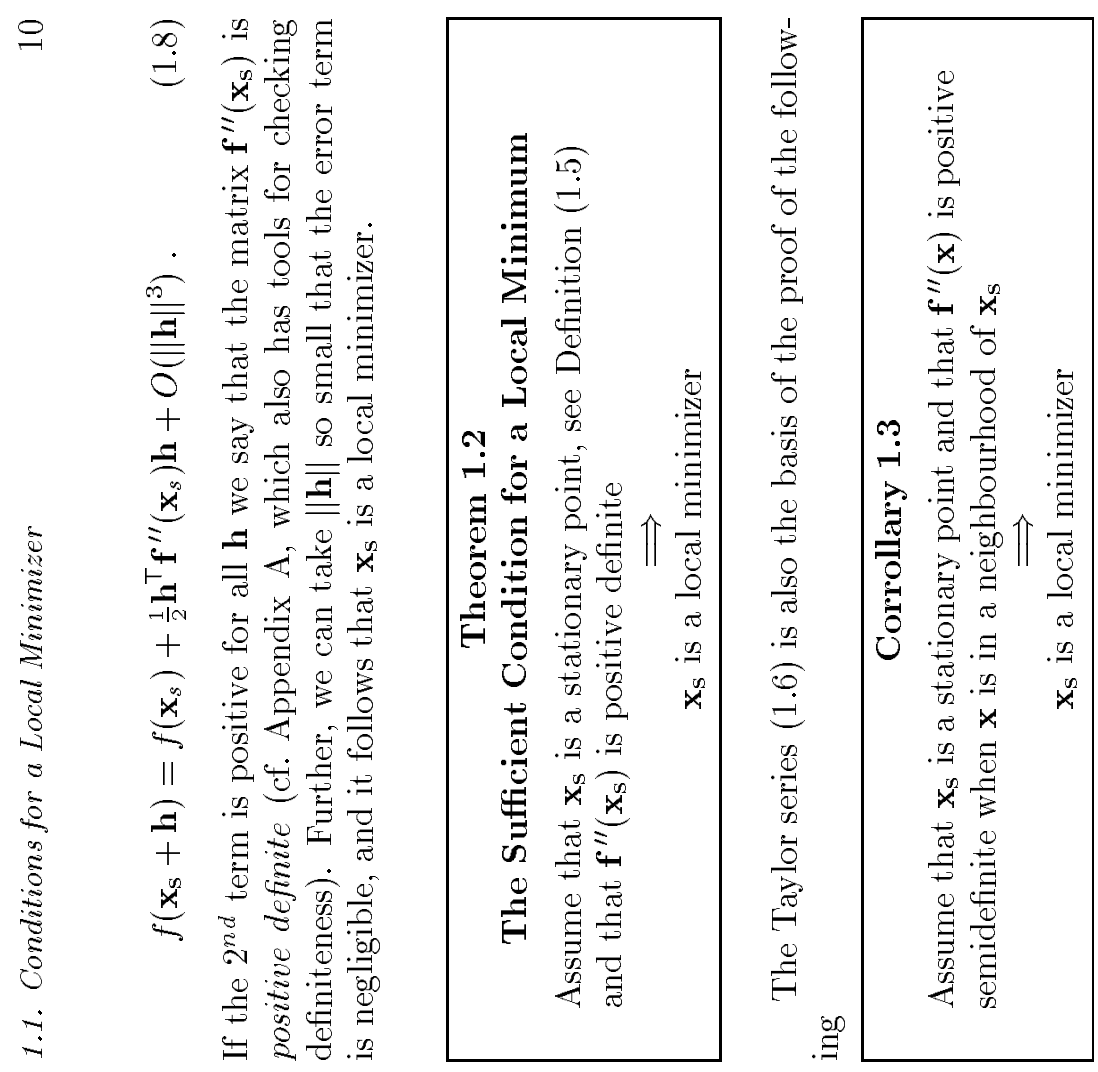

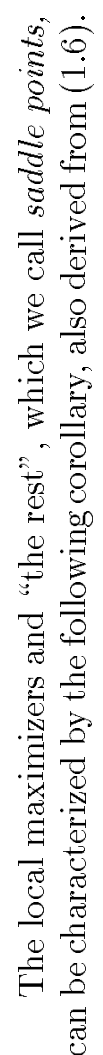
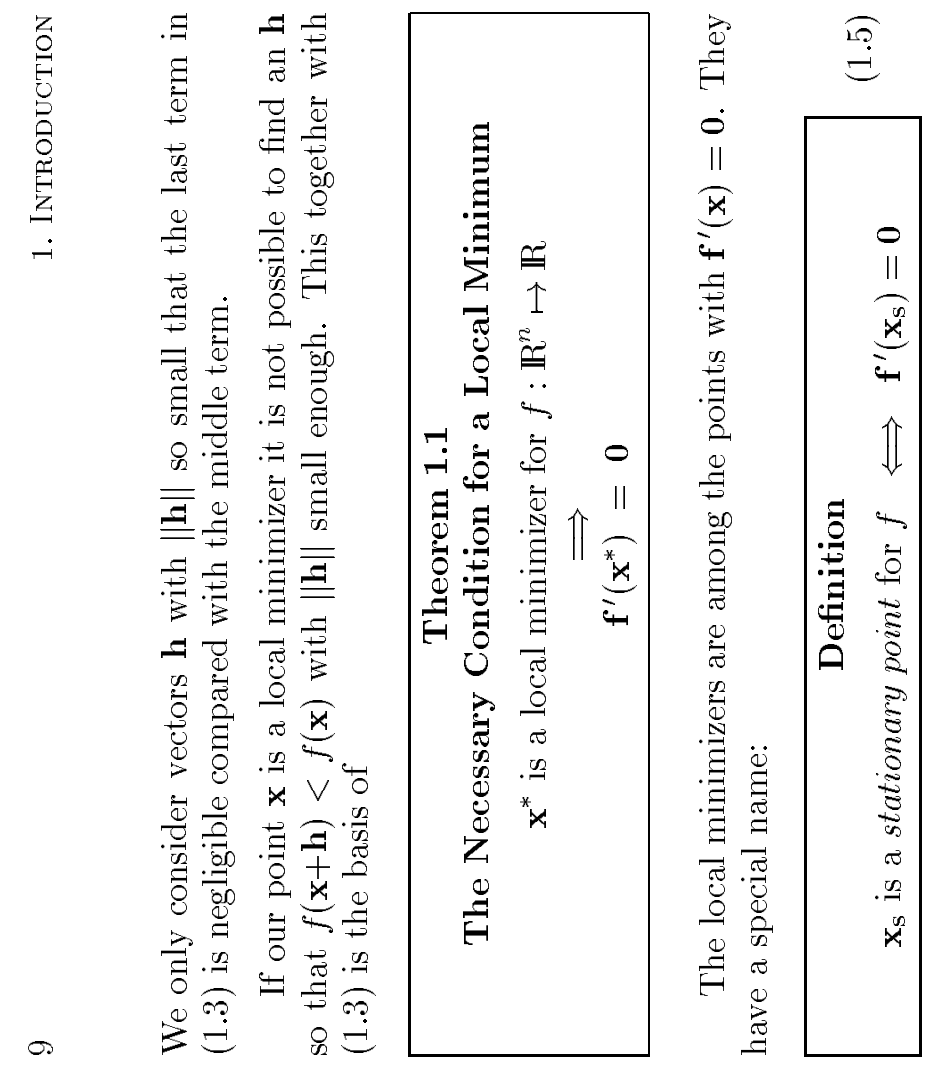

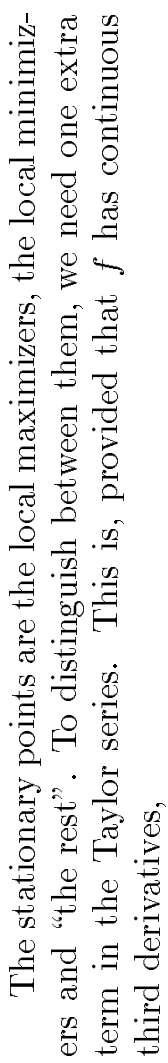

$\overparen{0}$

$\mp \quad \infty$

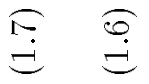

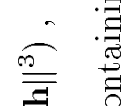

$\frac{\pi}{8}$

寄

.$\frac{\pi}{\pi}$

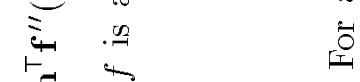

IN

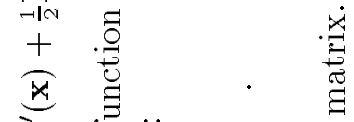

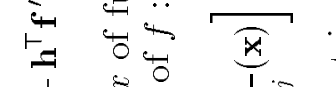

$x$
$+\infty$
$+\infty$

党

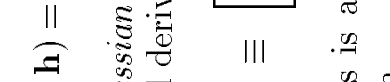

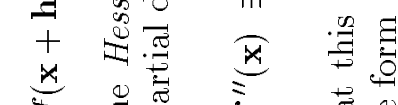

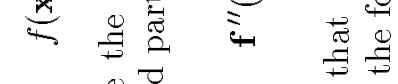



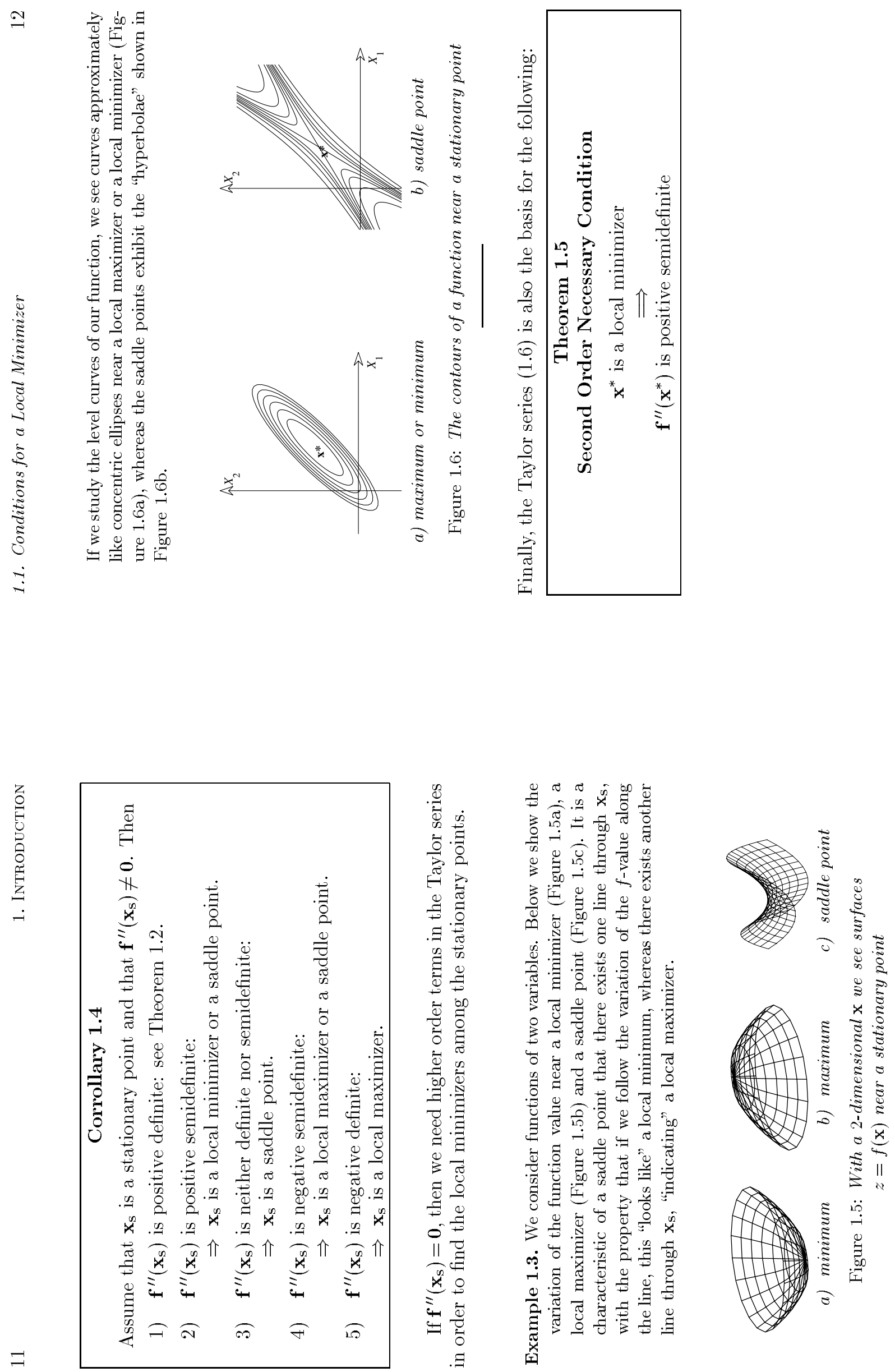

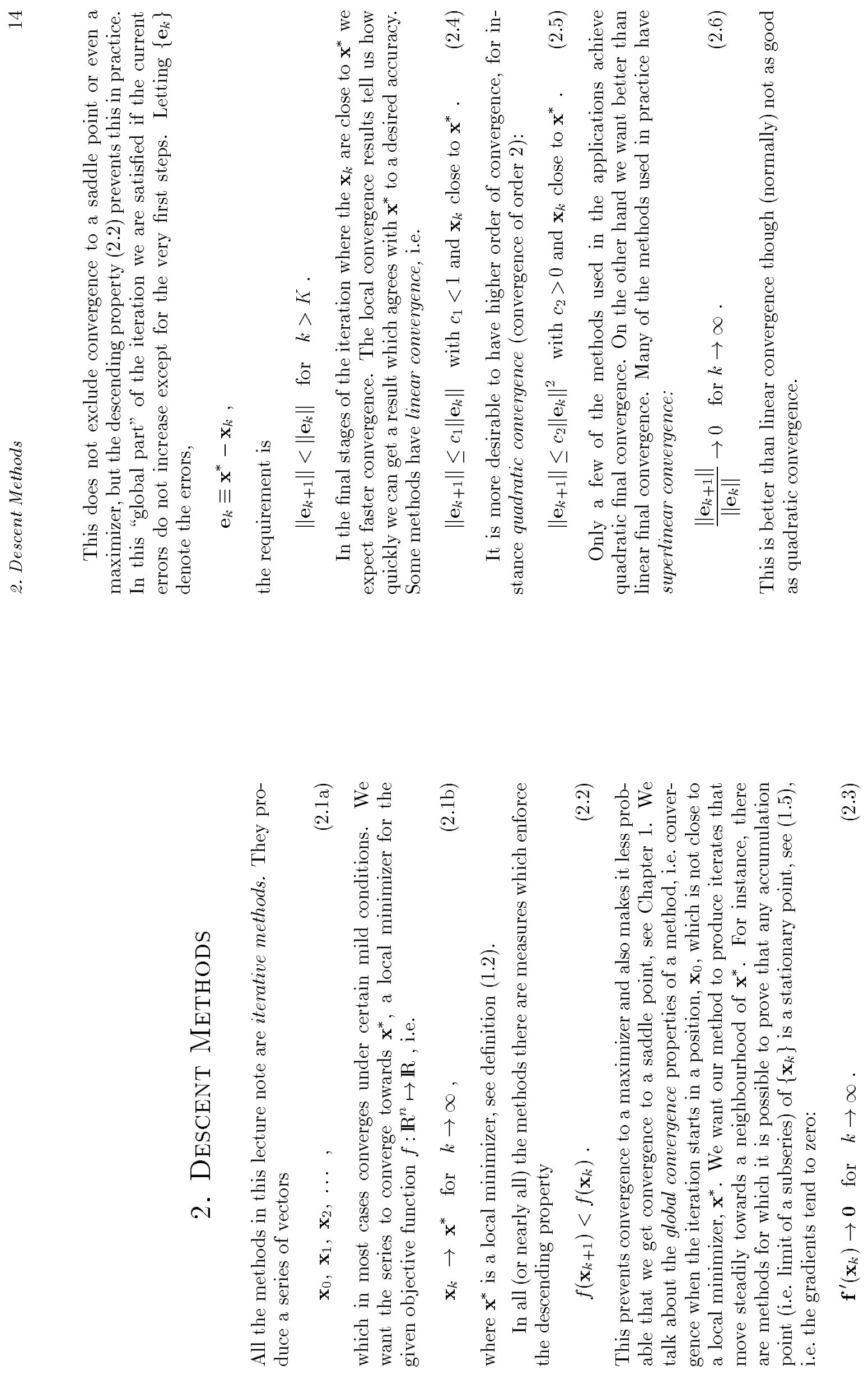

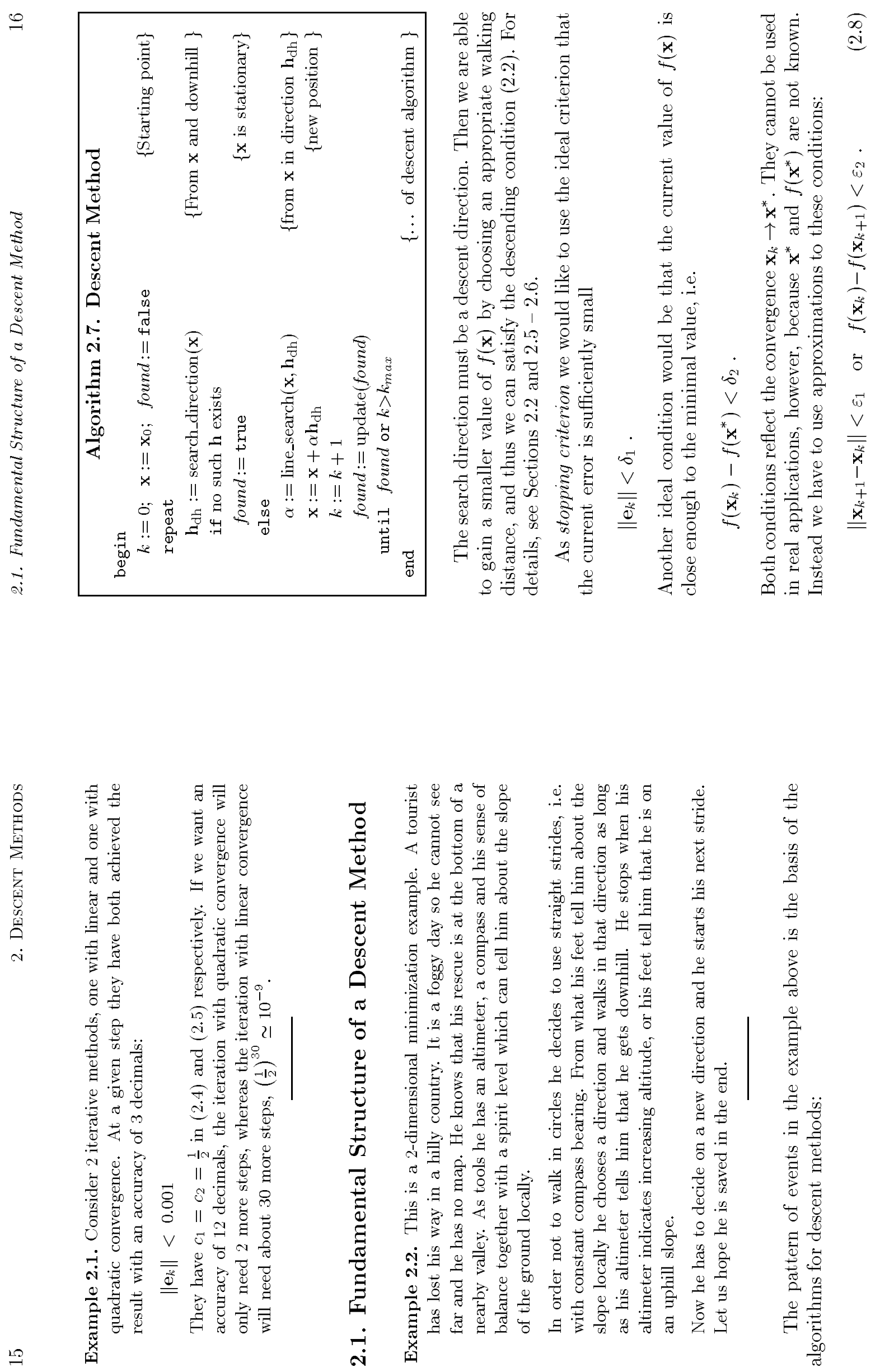


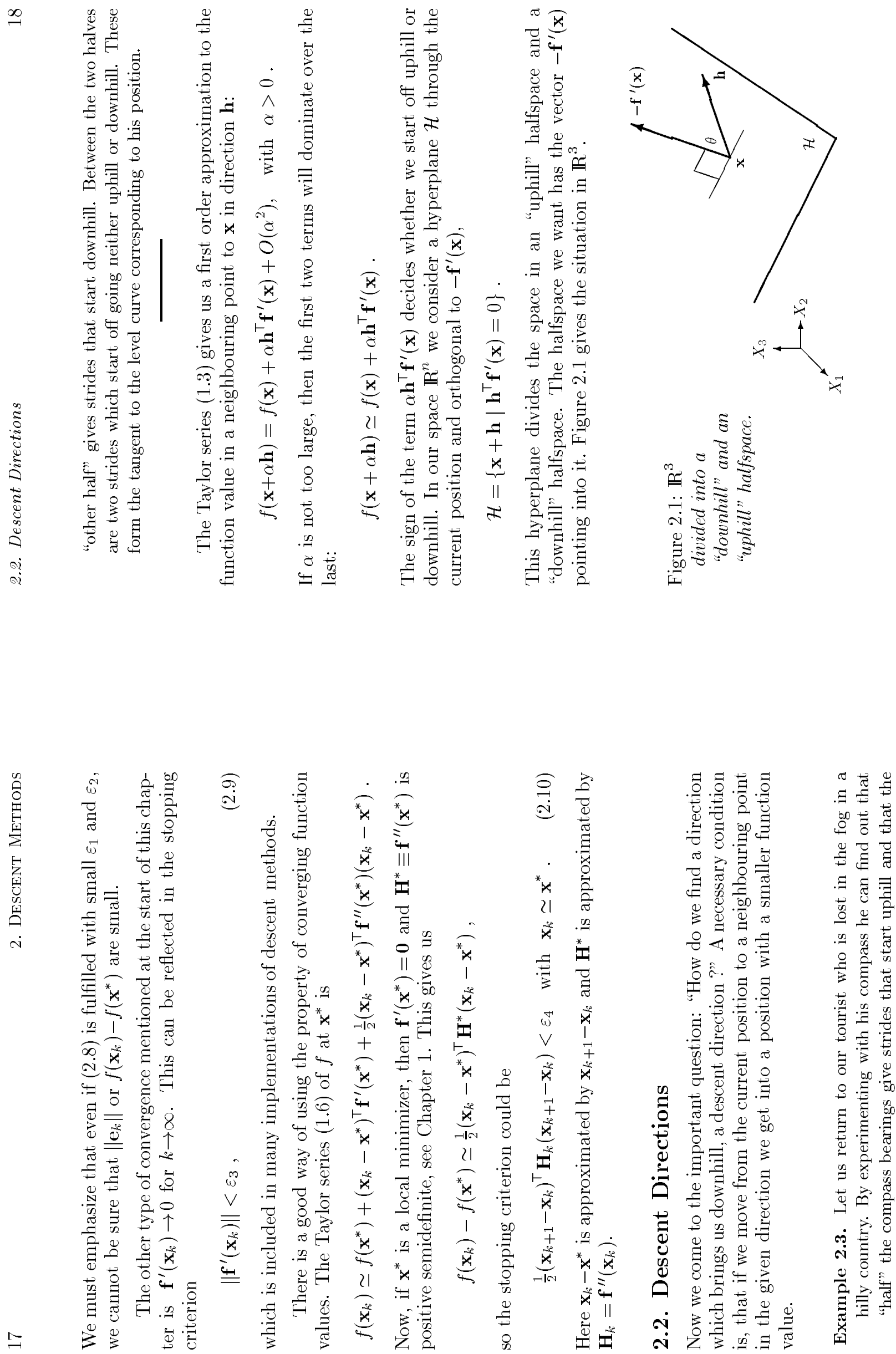


We now define a descent direction. This is a "downhill" direction, i.e. it is inside the "good" halfspace:

$\begin{aligned} \text { Definition } & \\ \mathbf{h} \text { is a descent direction from } \mathbf{x} & \Longleftrightarrow \mathbf{h}^{\top} \mathbf{f}^{\prime}(\mathbf{x})<0\end{aligned}$

A method based on condition (2.11) is a descent method.

In Figure 2.1 we have a descent direction $\mathbf{h}$, satisfying (2.11). We introduce the angle between $\mathbf{h}$ and $-\mathbf{f}^{\prime}(\mathbf{x})$

$$
\theta=L\left(\mathbf{h},-\mathbf{f}^{\prime}(\mathbf{x})\right) \quad \text { with } \quad \cos \theta=\frac{-\mathbf{h}^{\top} \mathbf{f}^{\prime}(\mathbf{x})}{\|\mathbf{h}\| \cdot\left\|\mathbf{f}^{\prime}(\mathbf{x})\right\|}
$$

We state a new condition on this angle,

Definition
An absolute descent method has search directions $\mathbf{h}_{k}$,
which satisfy
$\quad \theta<\frac{\pi}{2}-\mu$
for all $k$, with $\mu>0$ independent of $k$

The discussion above is concerned with the geometry in $\mathbb{R}^{3}$, and is easily seen to be valid also in $\mathbb{R}^{2}$. If the dimension $n$ is larger than 3 , we call $\theta$ "the pseudoangle between $\mathbf{h}$ and $-\mathbf{f}^{\prime}(\mathbf{x})$ ". In this way we can use (2.12) and (2.13), for all $n \geq 2$.

The restriction that $\mu$ must be constant in all the steps is necessary for the global convergence result we give in the next section.

\subsection{Descent Methods with Line Search}

When a descent direction has been determined, we have to decide how long the step in this direction should be. We perform a line search as indicated in Algorithm 2.7. First, we must be sure that the descending condition (2.2) is satisfied. Next, we must guard against the step being so short that our gain in function value diminishes. We study the variation of the objective function $f$ along the direction $\mathbf{h}$ from the current position $\mathbf{x}$

$$
\varphi(\alpha)=f(\mathbf{x}+\alpha \mathbf{h}), \quad \text { with fixed } \mathbf{x} \text { and } \mathbf{h} .
$$

From the Taylor series (1.6) it follows that

$$
\varphi(\alpha)=f(\mathbf{x})+\alpha \mathbf{h}^{\top} \mathbf{f}^{\prime}(\mathbf{x})+\frac{1}{2} \alpha^{2} \mathbf{h}^{\top} \mathbf{f}^{\prime \prime}(\mathbf{x}) \mathbf{h}+O\left(\alpha^{3}\right)
$$

and

$$
\varphi^{\prime}(0)=\mathbf{h}^{\top} \mathbf{f}^{\prime}(\mathbf{x})
$$

In Figure 2.2 we show an example of the variation of $\varphi(\alpha)$ with $\mathbf{h}$ as a descent direction. The descending condition (2.2) implies that we want to stop the line search with a value $\alpha_{\mathrm{S}}$ so that $\varphi\left(\alpha_{\mathrm{S}}\right)<\varphi(0)$. According to $(2.14)$ have $\varphi^{\prime}(0)<0$, but the figure shows that there is a risk that, if $\alpha$ is taken too large, then $\varphi(\alpha)>\varphi(0)$.

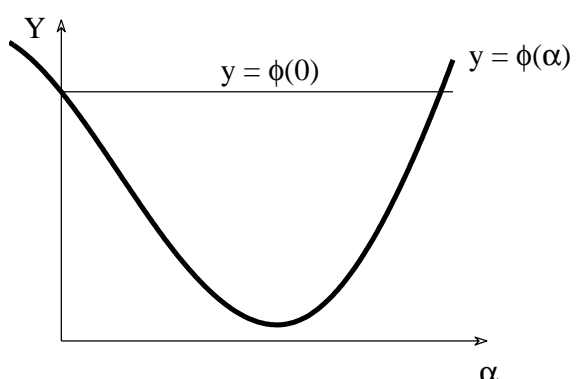

Figure 2.2: Variation of the cost function along the search line

To ensure that we get a useful decrease in $f$-value, we stop the search with a value $\alpha_{\mathrm{s}}$ which gives a $\varphi$-value below that of the line $y=\lambda(\alpha)$, indicated in Figure 2.3. This line goes through the starting point and has a slope which is a fraction of the slope of the starting tangent to the $\varphi$-curve: 


$$
\begin{aligned}
& \varphi\left(\alpha_{\mathrm{s}}\right) \leq \lambda\left(\alpha_{\mathrm{s}}\right), \quad \text { where } \\
& \lambda(\alpha)=\varphi(0)+\varrho \cdot \varphi^{\prime}(0) \cdot \alpha \quad \text { with } 0<\varrho<0.5 .
\end{aligned}
$$

The parameter $\varrho$ is normally small, 0.001 can be a good value. Condition (2.15) is needed in some convergence proofs.

We also want to ensure that the $\alpha$-value is not chosen too small. In Figure 2.3 we indicate a requirement, ensuring that the local slope is greater than the starting slope. More specificly,

$$
\varphi^{\prime}\left(\alpha_{\mathrm{s}}\right) \geq \beta \cdot \varphi^{\prime}(0) \quad \text { with } \varrho<\beta<1 .
$$

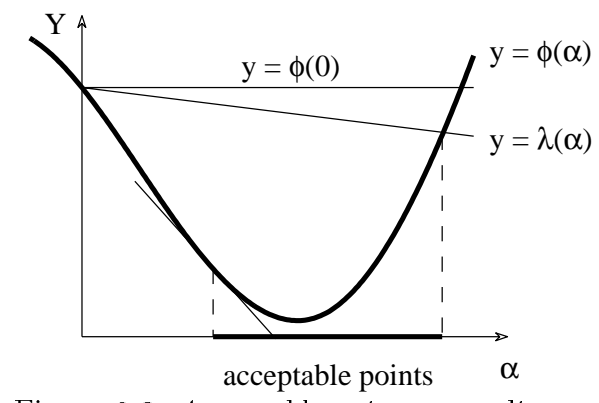

Figure 2.3: Acceptable points according to criteria (2.15) and (2.16)

Descent methods with line search governed by (2.15) plus (2.16) are normally convergent. Fletcher (1987), pp 26-30, has the proof of Theorem 2.1 below.

A possible outcome is that the method finds a stationary point $\left(\mathrm{x}_{k}\right.$ with $\mathbf{f}^{\prime}\left(\mathbf{x}_{k}\right)=\mathbf{0}$ ) and then it stops. Another possibility is that $f(\mathbf{x})$ is not bounded from below for $\mathbf{x}$ in the level set $\left\{\mathbf{x} \mid f(\mathbf{x})<f\left(\mathbf{x}_{0}\right)\right\}$ and the method may "fall into the hole". If neither of these occur, the method converges towards a stationary point. The method being a descent method often makes it converge towards a point which is not only a stationary point but also a local minimizer.

\section{Theorem 2.1}

Consider an absolute descent method following Algorithm 2.7 with search directions according to (2.12) and (2.13) and with line search controlled by (2.15) and (2.16).

If $\mathbf{f}^{\prime}(\mathbf{x})$ exists and is uniformly continuous on the level set $\left\{\mathbf{x} \mid f(\mathbf{x})<f\left(\mathbf{x}_{0}\right)\right\}$, then for $k \rightarrow \infty$ :

either $\mathbf{f}^{\prime}\left(\mathbf{x}_{k}\right)=\mathbf{0}$ for some $k$

or $f\left(\mathbf{x}_{k}\right) \rightarrow-\infty$

or $\quad \mathbf{f}^{\prime}\left(\mathbf{x}_{k}\right) \rightarrow \mathbf{0}$

A line search as described above is often called a soft line search because of its liberal stopping criteria, (2.15) and (2.16). In contrast to this there are variants which we call "exact line searches", exact in the sense that we seek an approximation to a local minimizer for $\varphi(\alpha)$, i.e.

$$
\alpha_{\mathbf{e}}=\operatorname{argmin}_{\alpha>0} f(\mathbf{x}+\alpha \mathbf{h}) \text { for fixed } \mathbf{x} \text { and } \mathbf{h} .
$$

A necessary condition on $\alpha_{\mathrm{e}}$ is $\varphi^{\prime}\left(\alpha_{\mathrm{e}}\right)=0$. We have $\varphi^{\prime}(\alpha)=$ $\mathbf{h}^{\top} \mathbf{f}^{\prime}(\mathbf{x}+\alpha \mathbf{h})$ and this shows that either $\mathbf{f}^{\prime}\left(\mathbf{x}+\alpha_{\mathbf{e}} \mathbf{h}\right)=\mathbf{0}$, which is a perfect result (we have found a stationary point for $f$ ), or if $\mathbf{f}^{\prime}\left(\mathbf{x}+\alpha_{\mathrm{e}} \mathbf{h}\right) \neq \mathbf{0}$, then $\varphi^{\prime}\left(\alpha_{\mathrm{e}}\right)=0$ leads to:

$$
\mathbf{f}^{\prime}\left(\mathbf{x}+\alpha_{\mathbf{e}} \mathbf{h}\right) \perp \mathbf{h} .
$$

This shows that the exact line search will stop at a point where the local gradient is orthogonal to the search direction.

Example 2.4. A "divine power" with a radar set follows the movements of our wayward tourist. He has decided to continue in a given direction, until his feet or his altimeter tells him that he starts to go uphill. The "divine power" can see that he stops where the given direction is tangent to a local contour. This is equivalent to the orthogonality mentioned in (2.18). 

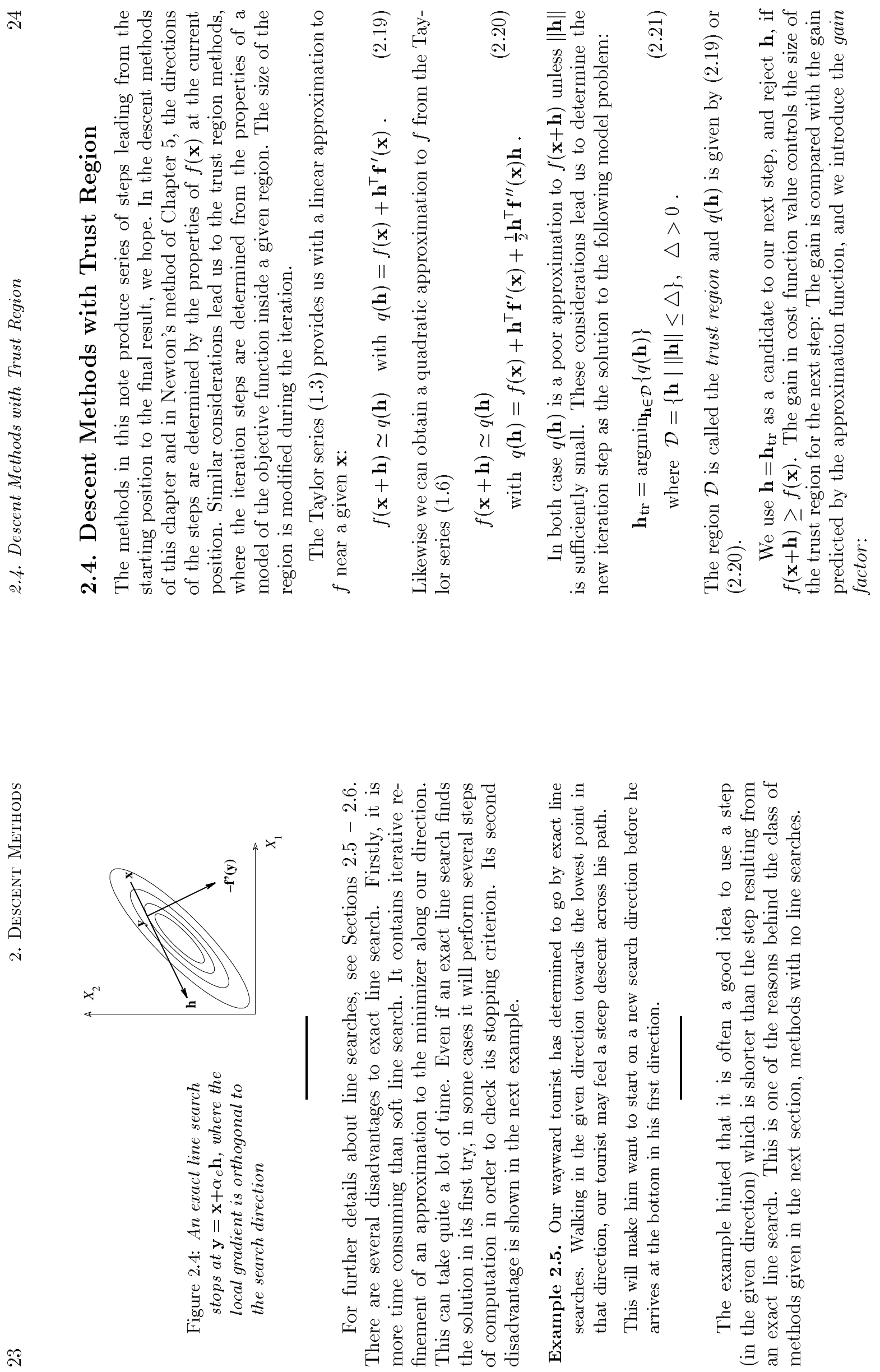

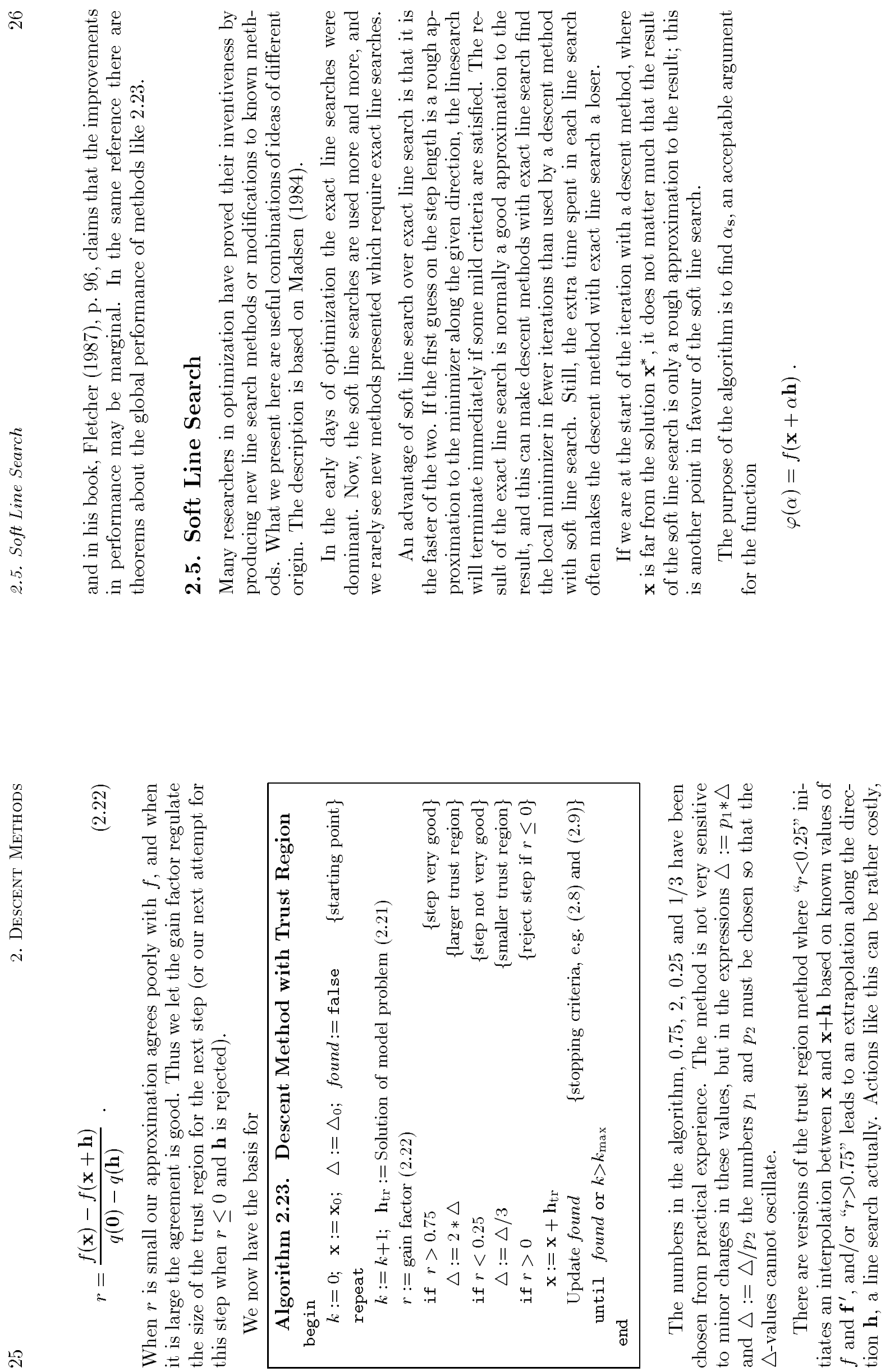
The acceptability is decided by the criteria (2.15),

$$
\begin{aligned}
& \varphi\left(\alpha_{\mathrm{S}}\right) \leq \lambda\left(\alpha_{\mathrm{S}}\right), \quad \text { where } \\
& \lambda(\alpha)=\varphi(0)+\varrho \cdot \varphi^{\prime}(0) \cdot \alpha \quad \text { with } \quad 0<\varrho<0.5
\end{aligned}
$$

and (2.16),

$$
\varphi^{\prime}\left(\alpha_{\mathrm{S}}\right) \geq \beta \cdot \varphi^{\prime}(0) \text { with } \varrho<\beta<1 .
$$

These two criteria express the demands that $\alpha_{\mathrm{s}}$ must be sufficiently small to give a useful decrease in the objective function, and sufficiently large to ensure that we have left the starting tangent of the curve $y=\varphi(\alpha)$ for $\alpha \geq 0$; cf. Figure 2.3.

The algorithm has two parts. First we find an interval $[a, b]$ that contains acceptable points, see figure 2.5:

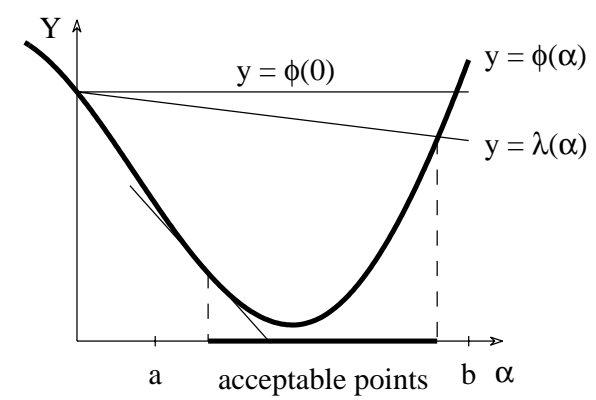

Figure 2.5: Interval $[a, b]$ containing acceptable points

In the second part of the algorithm we successively reduce the interval: We find a point $\alpha$ in the strict interior of $[a, b]$. If both conditions (2.24) are satisfied by this $\alpha$-value, then we are finished $\left(\alpha_{\mathrm{s}}=\alpha\right)$. Otherwise, the reduced interval is either $[a, b]:=[a, \alpha]$ or $[a, b]:=[\alpha, b]$, where the choice is made so that the reduced $[a, b]$ contains acceptable points.

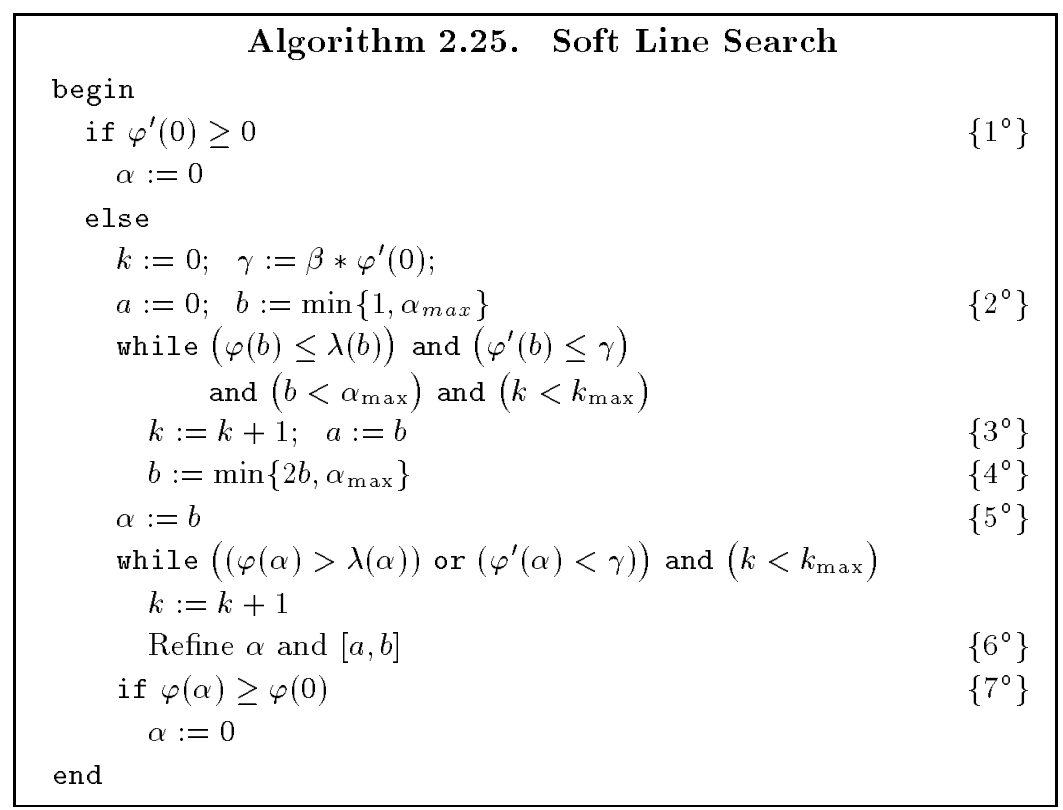

We have the following remarks:

$1^{\circ}$ If $\mathbf{x}$ is a stationary point $\left(\mathbf{f}^{\prime}(\mathbf{x})=\mathbf{0} \Rightarrow \varphi^{\prime}(0)=0\right)$ or $\mathbf{h}$ is not downhill, then we do nothing.

$2^{\circ}$ The initial choice $b=1$ is used because in many optimization methods (e.g. Newton's method in Chapter 5) $\alpha=1$ is a very good guess in the final steps of the iteration. The upper bound $\alpha_{\max }$ must be supplied by the user. It acts as a guard against an infinite loop if $f$ is unbounded.

$3^{\circ}$ We are to the left of a minimum and update the left hand end of the interval $[a, b]$. 

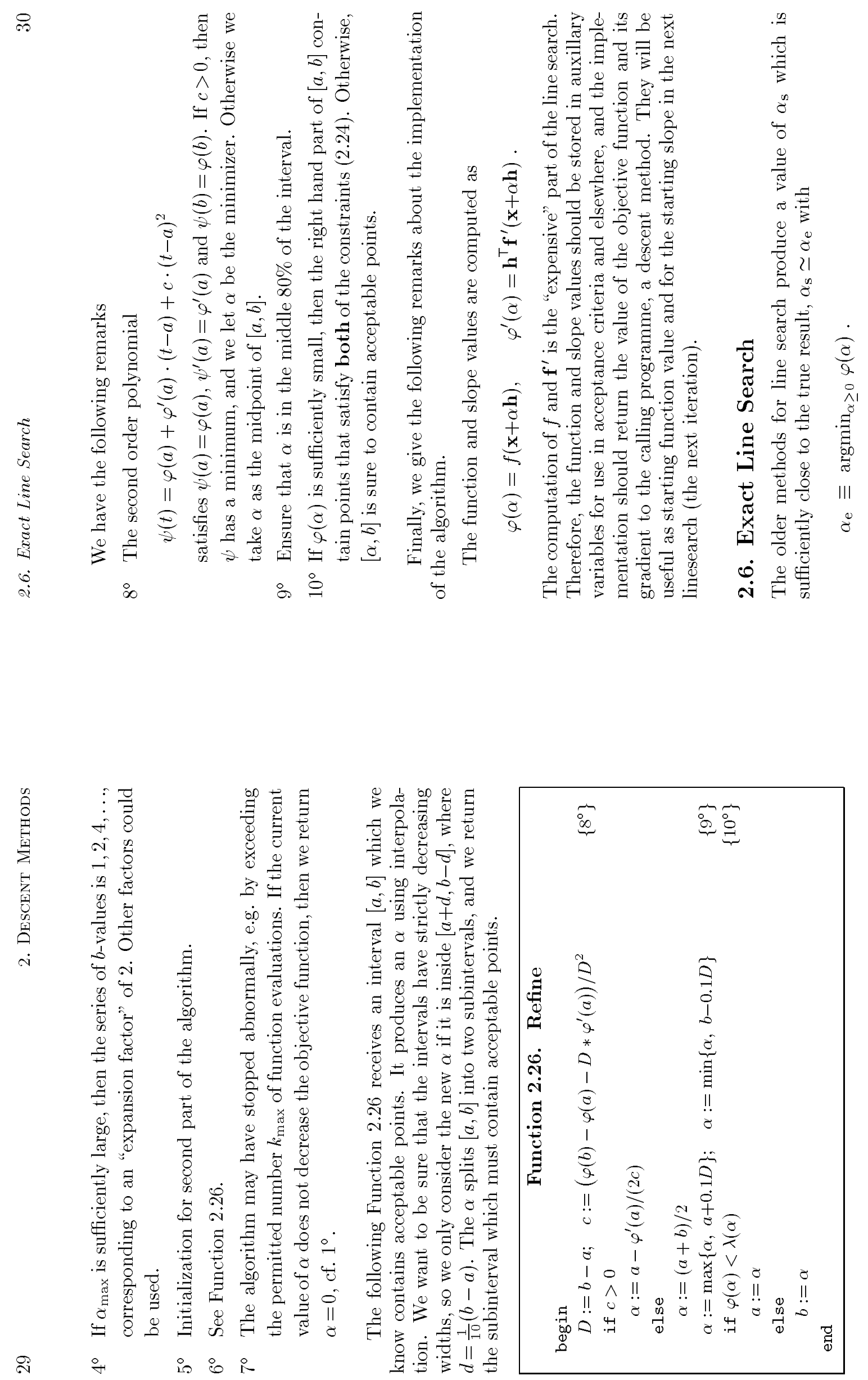

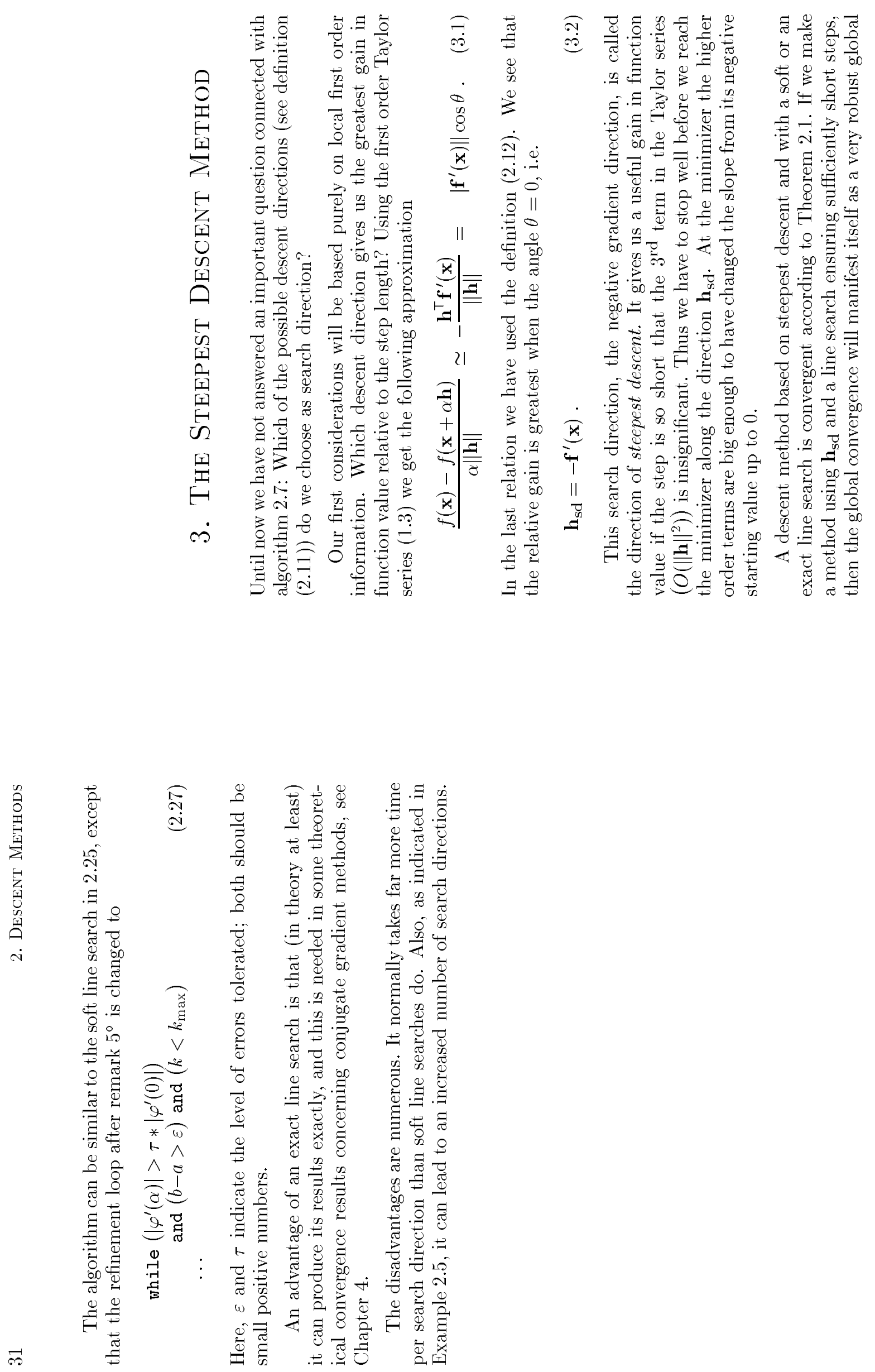

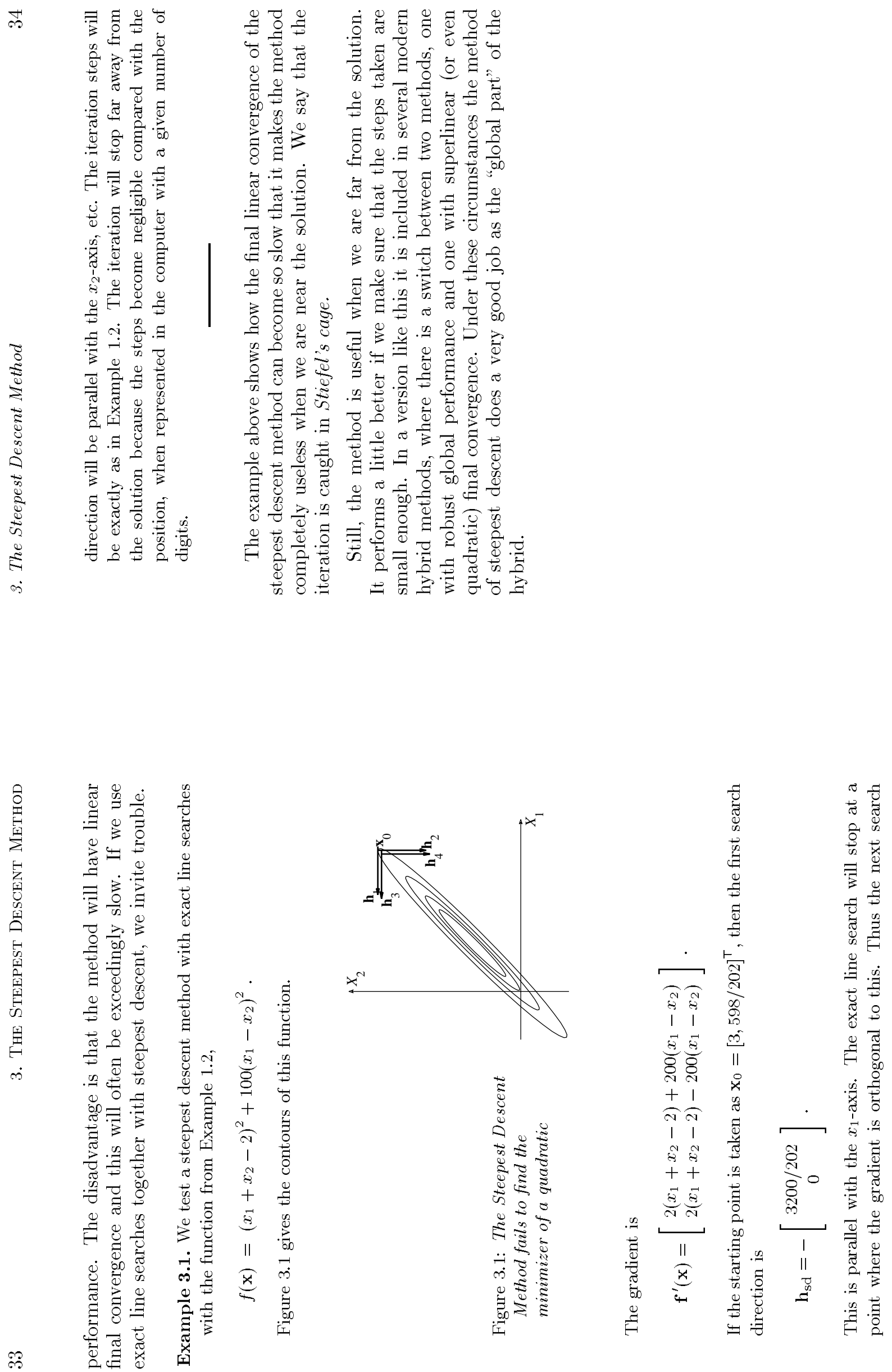


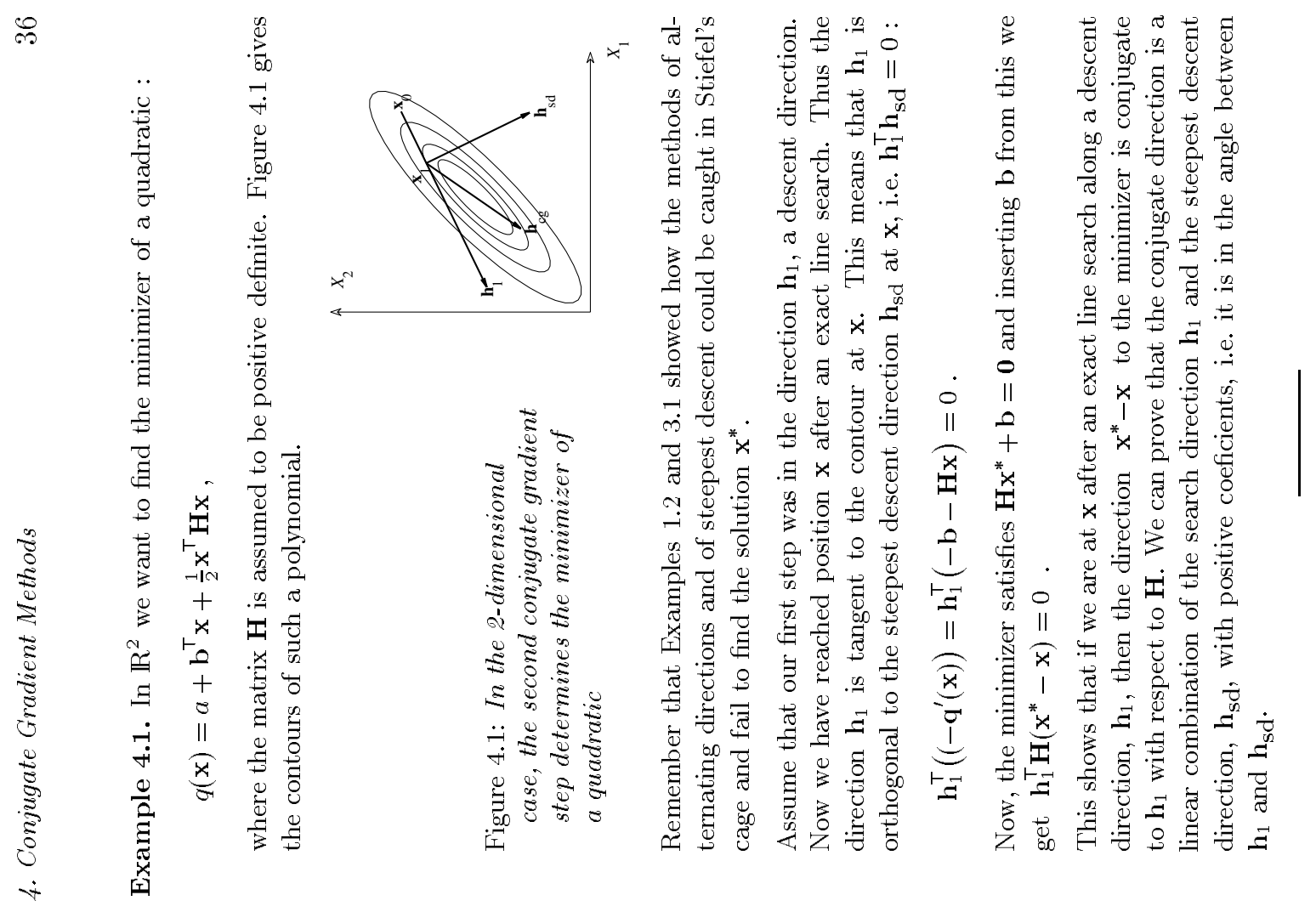

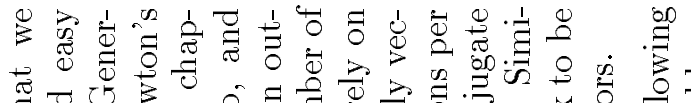

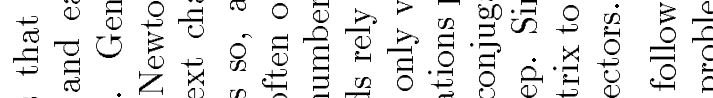

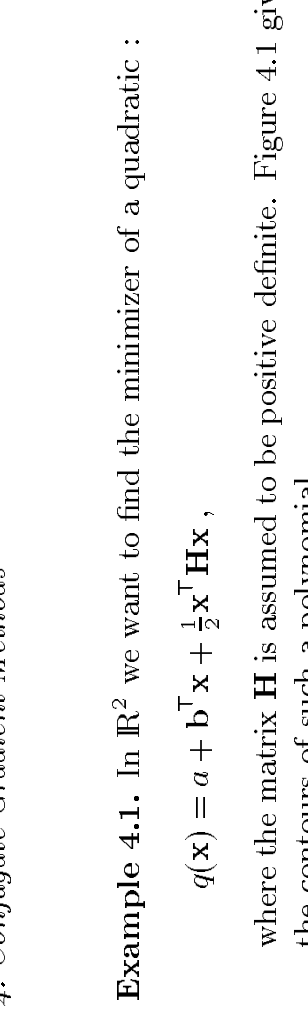

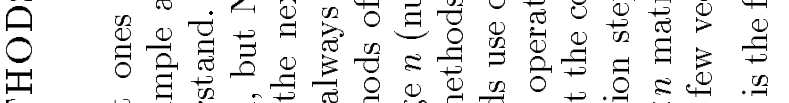

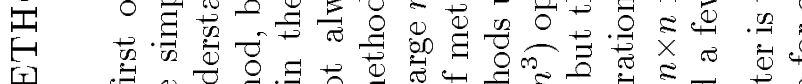

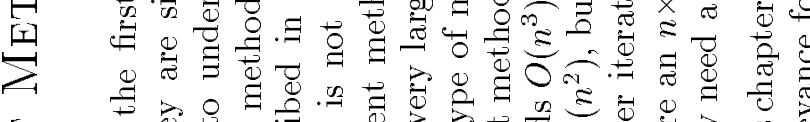

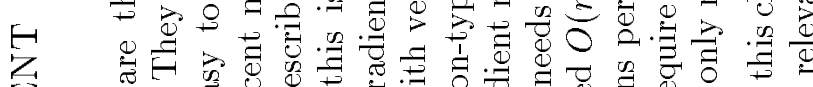

I

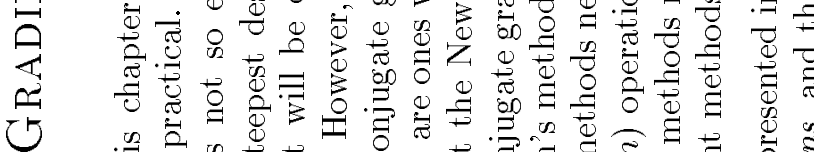

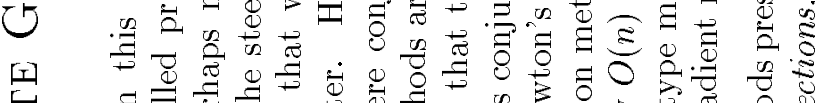

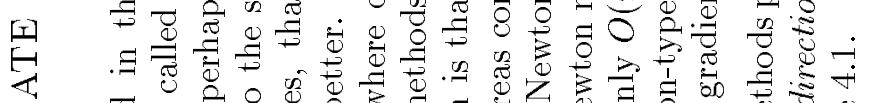

万人

Ð

芒

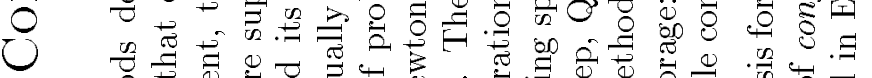

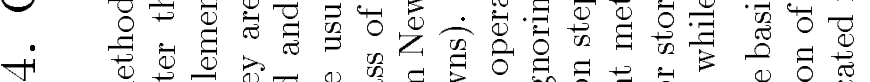

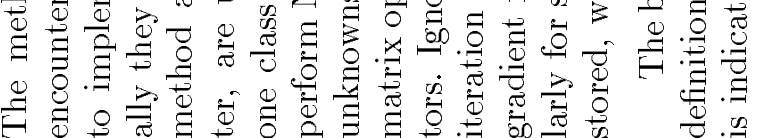

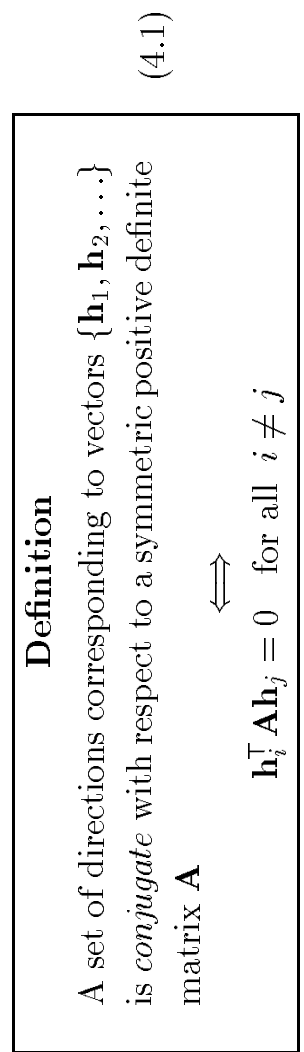



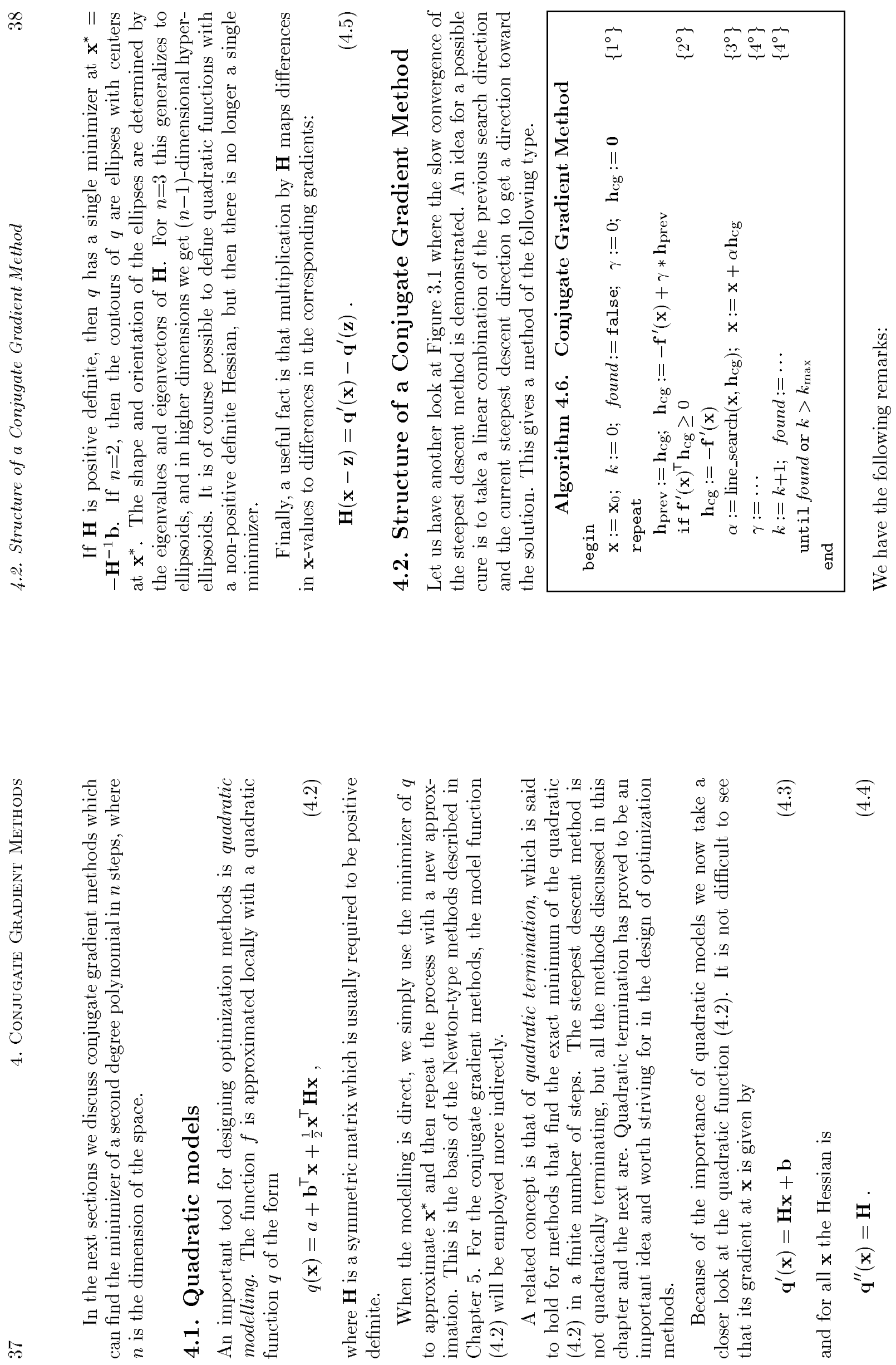

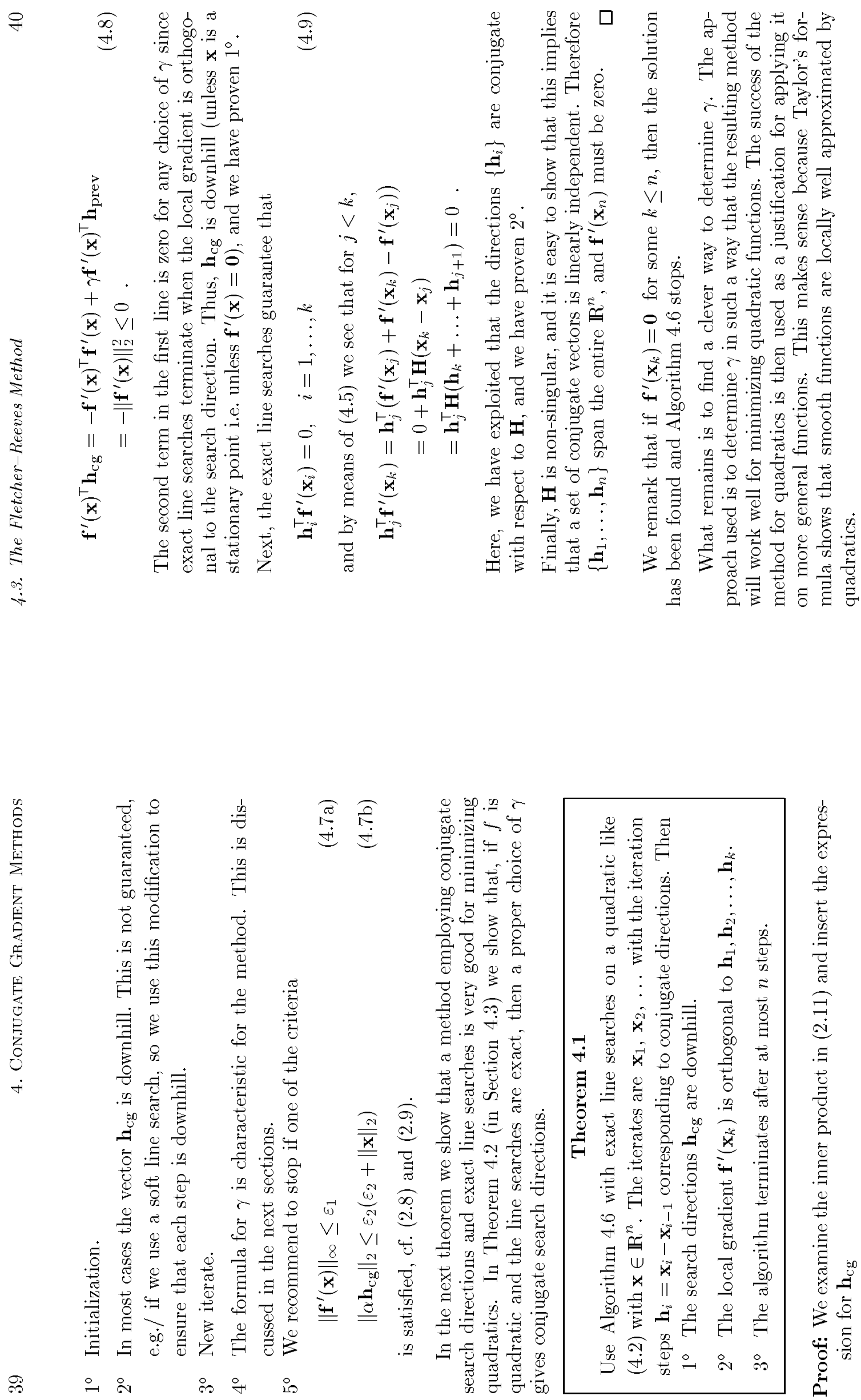


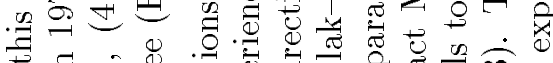

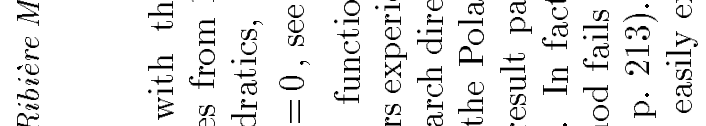

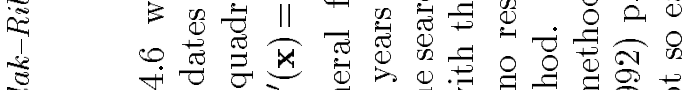
ฉ

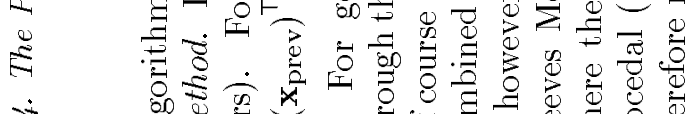

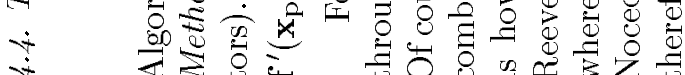

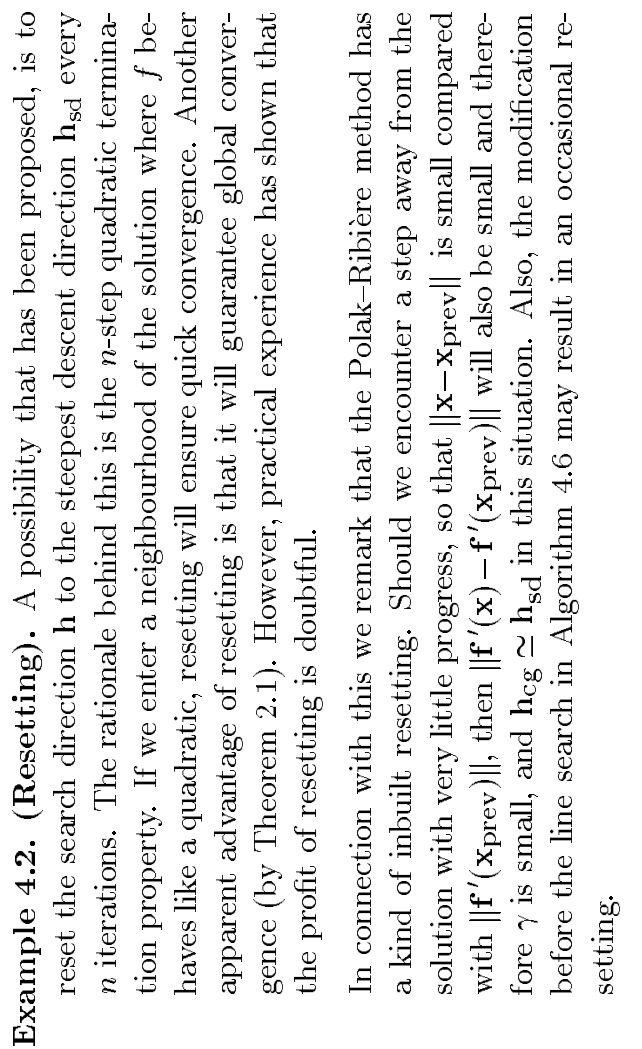

(1)

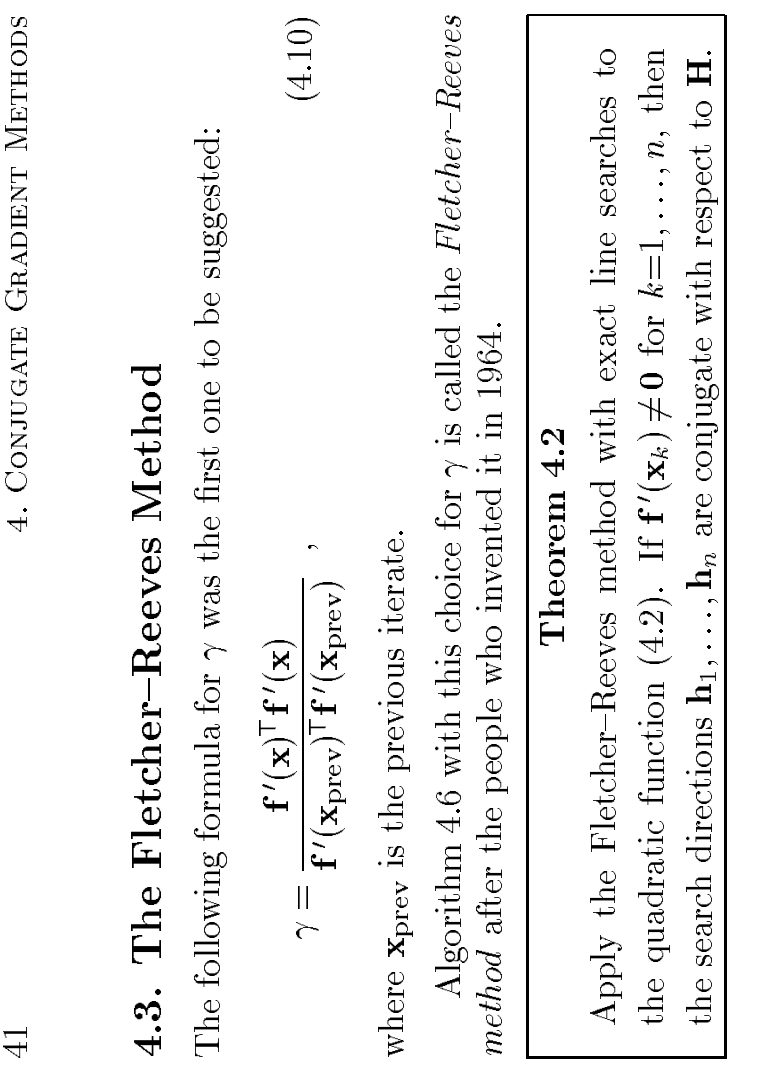

$\square$

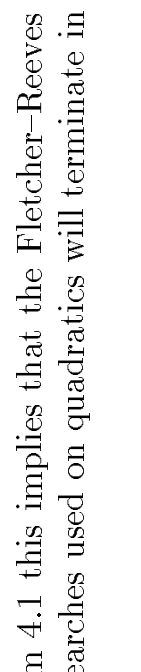

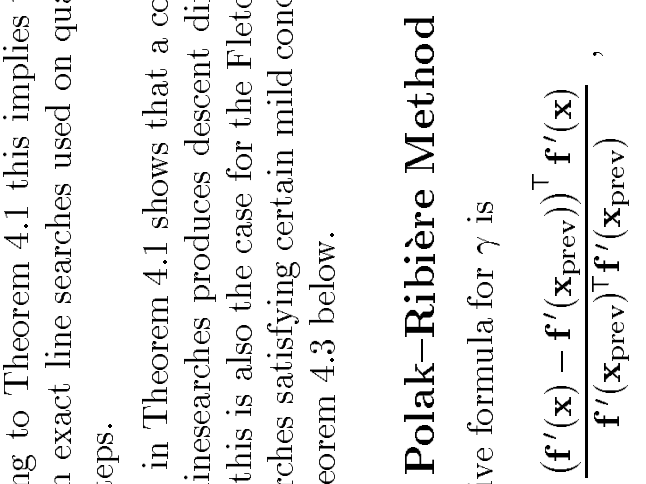

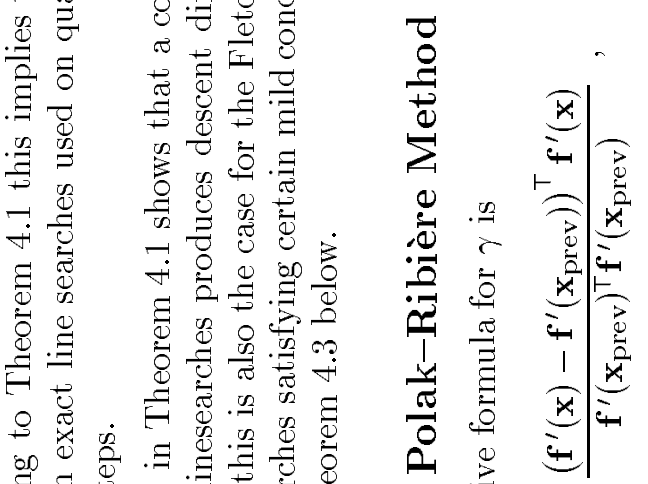

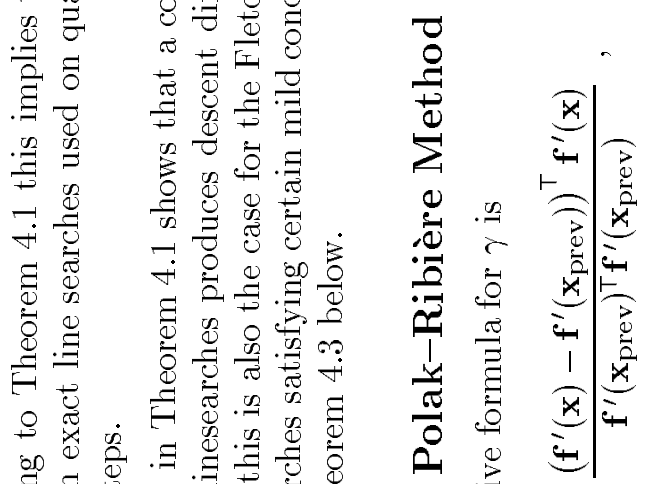

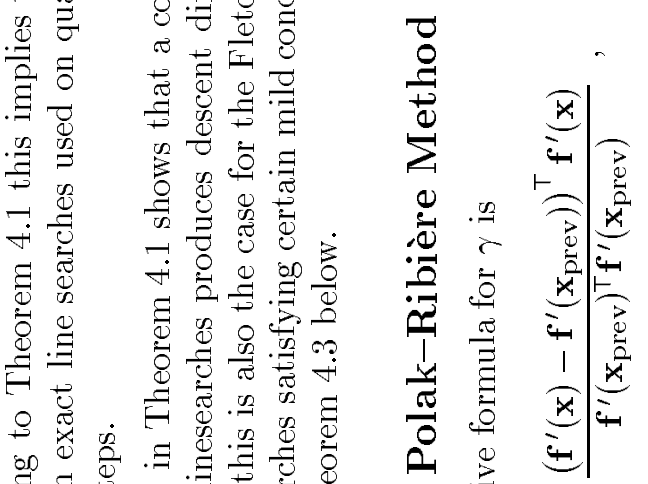
So

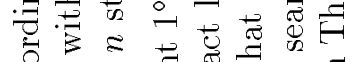

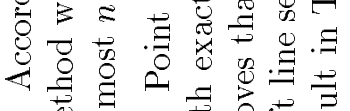

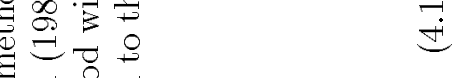

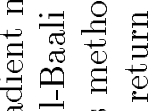
क्ष प के

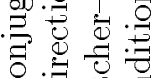

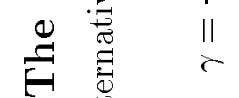



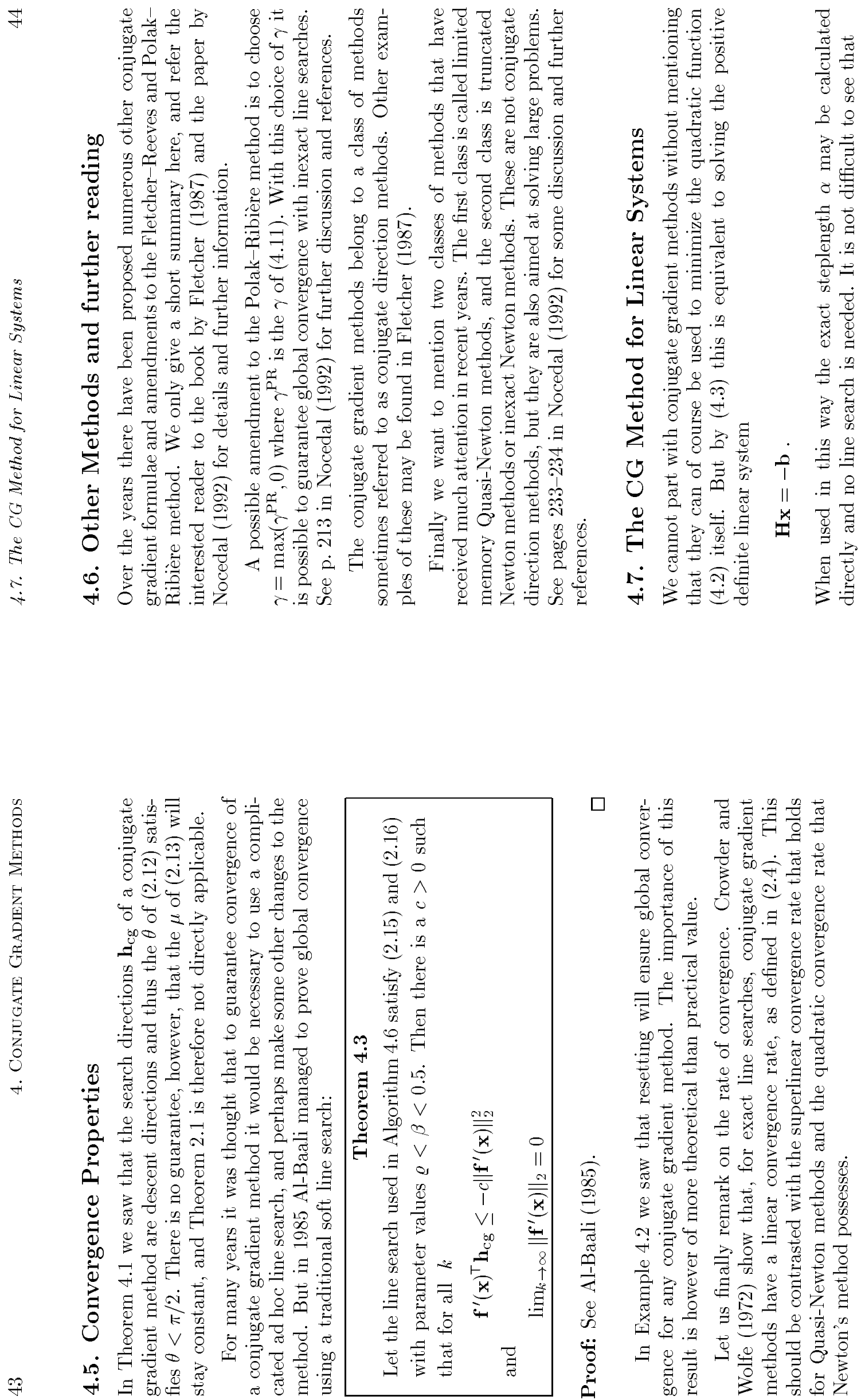


$$
\alpha=\frac{-\mathbf{h}_{\mathrm{cg}}^{\top} \mathbf{H}(\mathbf{x}+\mathbf{b})}{\mathbf{h}_{\mathrm{cg}}^{\top} \mathbf{H} \mathbf{h}_{\mathrm{cg}}} .
$$

The Fletcher-Reeves and the Polak-Ribière formulae are equivalent in this setting, and the resulting method is called the conjugate gradient method for linear systems. Its study is a whole subject in itself, within the field of numerical linear algebra.

One situation where this method may be preferrable is when the system to be solved is large and sparse. Since the conjugate gradient method only needs matrix-vector multiplications it can then be much cheaper than a direct method, e.g. Gaussian elimination.

\subsection{Implementation}

To implement a conjugate gradient algorithm in a computer program, some decisions must be made. Of course we need to choose a formula for $\gamma$. Here the Polak-Ribière formula is recommended.

We also need to specify the exactness of the line search. For Newton-type methods it is usually recommended that the line search be quite soft, so for the line search in Algorithm 2.25 it is common to choose the parameter values $\varrho=0.01$ and $\beta=0.9$. For conjugate gradient methods experience dictates that a line search with stricter tolerances be used, say $\varrho=0.01$ and $\beta=0.1$. In addition we have to specify the stopping criterion. Here (2.9) is recommended. We do not have acces to $\mathbf{f}^{\prime \prime}\left(\mathbf{x}_{k}\right)$ and therefore cannot use (2.10). For methods with a fast convergence rate, (2.8) may be quite satisfactory, but its use for conjugate gradient methods must be discouraged because their final convergence rate is only linear.

Finally some remarks on the storage of vectors. The FletcherReeves method may be implemented using three $n$-vectors of storage, $\mathbf{x}, \mathrm{g}$ and $\mathrm{h}$. If these contain $\mathbf{x}, \mathbf{f}^{\prime}(\mathbf{x})$ and $\mathbf{h}_{\text {prev }}$ at the beginning of the current iteration step, we may overwrite $\mathbf{h}$ with $\mathbf{h}_{\mathrm{cg}}$ and during the line search we overwrite $\mathbf{x}$ with $\mathbf{x}+\alpha \mathbf{h}_{\mathrm{cg}}$ and $\mathrm{g}$ with $\mathbf{f}^{\prime}\left(\mathbf{x}+\alpha \mathbf{h}_{\mathrm{cg}}\right)$.
Before overwriting the gradient, we find $\mathbf{f}^{\prime}(\mathbf{x})^{\top} \mathbf{f}^{\prime}(\mathbf{x})$ for use in the denominator in (4.10) on the next iteration. For the Polak-Ribière method we need acces to $\mathbf{f}^{\prime}(\mathbf{x})$ and $\mathbf{f}^{\prime}\left(\mathbf{x}_{\text {prev }}\right)$ simultaneously, and thus four vectors are required, say $\mathrm{x}, \mathrm{g}$, gnew and $\mathrm{h}$.

Example 4.3. Rosenbrock's function,

$$
f(\mathrm{x})=100\left(x_{2}-x_{1}^{2}\right)^{2}+\left(1-x_{1}\right)^{2},
$$

is widely used for testing optimization algorithms. Figure 4.2 shows level curves for this function (and illustrates, why it is sometimes called the "banana function").

The function has one minimizer $\mathrm{x}^{*}=[1,1]^{\top}$ with $f\left(\mathrm{x}^{*}\right)=0$, and there is a "valley" with sloping bottom following the parabola $x_{2}=x_{1}^{2}$. Most optimization algorithms will try to follow this valley. Thus, we will need a considerable amount of iteration steps if we take $\mathrm{x}_{0}$ in the 2 nd quadrant.

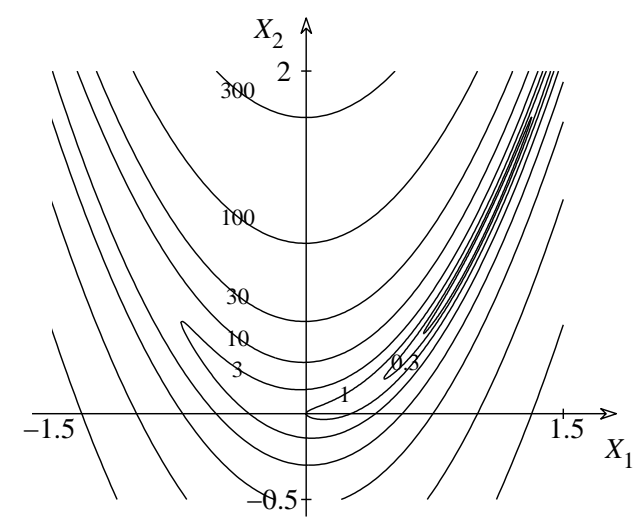

Figure 4.2: Contours of Rosenbrock's function

Below we give the number of iteration steps and evaluations of $f(\mathrm{x})$ and $\mathbf{f}^{\prime}(\mathrm{x})$ when applying Algorithm 4.6 on this function. In all cases 
we use the starting point $\mathbf{x}_{0}=[-1.2,1]^{\top}$, and stopping criteria given by $\varepsilon_{1}=10^{-8}, \varepsilon_{2}=10^{-12}$ in (4.7). In case of exact line search we use $\tau=10^{-6}, \varepsilon=10^{-6}$ in $(2.27)$, while we take $\beta=10^{-1}, \varrho=10^{-2}$ in Algorithm 2.25 for soft line search.

\begin{tabular}{|c|c|c|c|}
\hline Method & Line search & \# it. steps & \# fct. evals \\
\hline Fletcher-Reeves & exact & 118 & 1429 \\
Fletcher-Reeves & soft & 249 & 628 \\
\hline Polak-Ribière & exact & 24 & 266 \\
Polak-Ribière & soft & 45 & 130 \\
\hline
\end{tabular}

Thus, in this case the Polak-Ribière method with soft line search performs best. Below we give the iterates (cf. Figure 4.2) and the values of $f\left(\mathbf{x}_{k}\right)$ and $\left\|\mathbf{f}^{\prime}\left(\mathbf{x}_{k}\right)\right\|_{\infty}$; note the logarithmic ordinate axis.
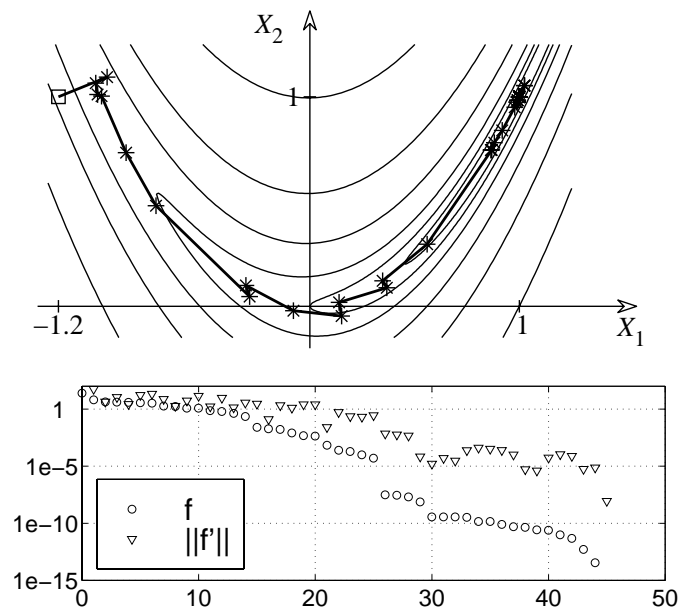

Figure 4.3: Polak-Ribière method with soft line search applied to Rosenbrock's function.

Top: iterates $\mathrm{x}_{k} . \quad$ Bottom: $f\left(\mathbf{x}_{k}\right)$ and $\left\|\mathbf{f}^{\prime}\left(\mathbf{x}_{k}\right)\right\|_{\infty}$.

\section{Newton-Type Methods}

In this chapter we consider a class of methods for unconstrained optimization which are based on Newton's method. This class is called Quasi-Newton methods. In order to explain these methods we first describe Newton's method for unconstrained optimization in detail. Newton's method leads to another kind of methods known as Damped Newton Methods, which will also be presented.

Finally we get to the Quasi-Newton methods. This class includes some of the best methods on the market for solving the unconstrained optimization problem.

\subsection{Newton's Method}

Newton's method forms the basis of all Quasi-Newton methods. It is widely used for solving systems of non-linear equations, and until recently it was also widely used for solving unconstrained optimization problems. As it will appear, the two problems are closely related.

Example 5.1. In Example 1.2 we saw the method of alternating directions fail to find the minimizer of a simple quadratic in two dimensions and in Example 3.1 we saw the steepest descent method fail on the same quadratic. In Chapter 4 we saw that the conjugate gradient methods finds the minimizer of a quadratic in $n$ steps ( $n$ being the dimension of the space), in two steps in Example 4.1.

Newton's method can find the minimizer of a quadratic in $n$ dimensional space in one step. This follows from equation (5.2) below.

Figure 5.1 gives the contours of our 2-dimensional quadratic together with (an arbitrary) $\mathrm{x}_{0} \cdot \mathrm{x}_{1}$ and the minimizer $\mathrm{x}^{*}$, marked by $*$. 

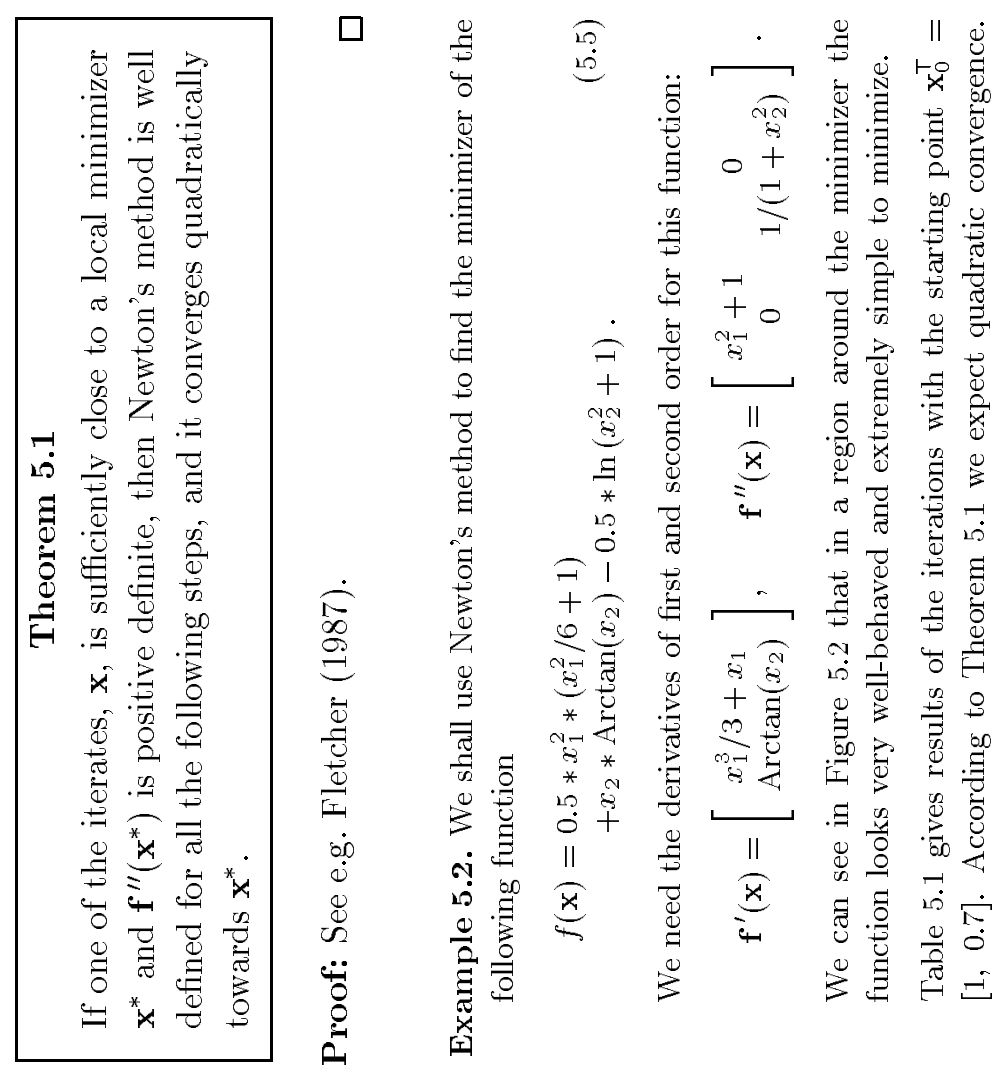

要
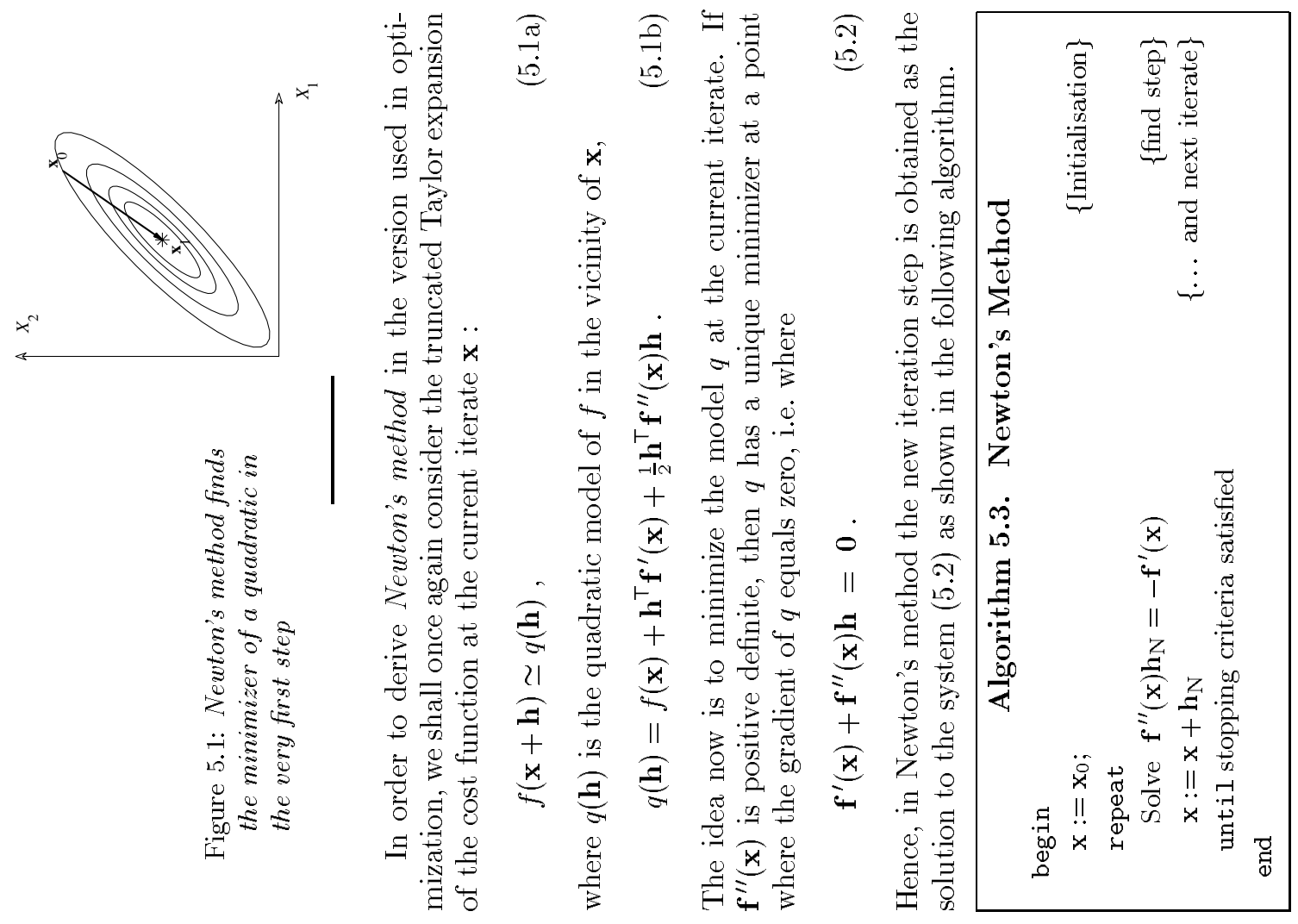

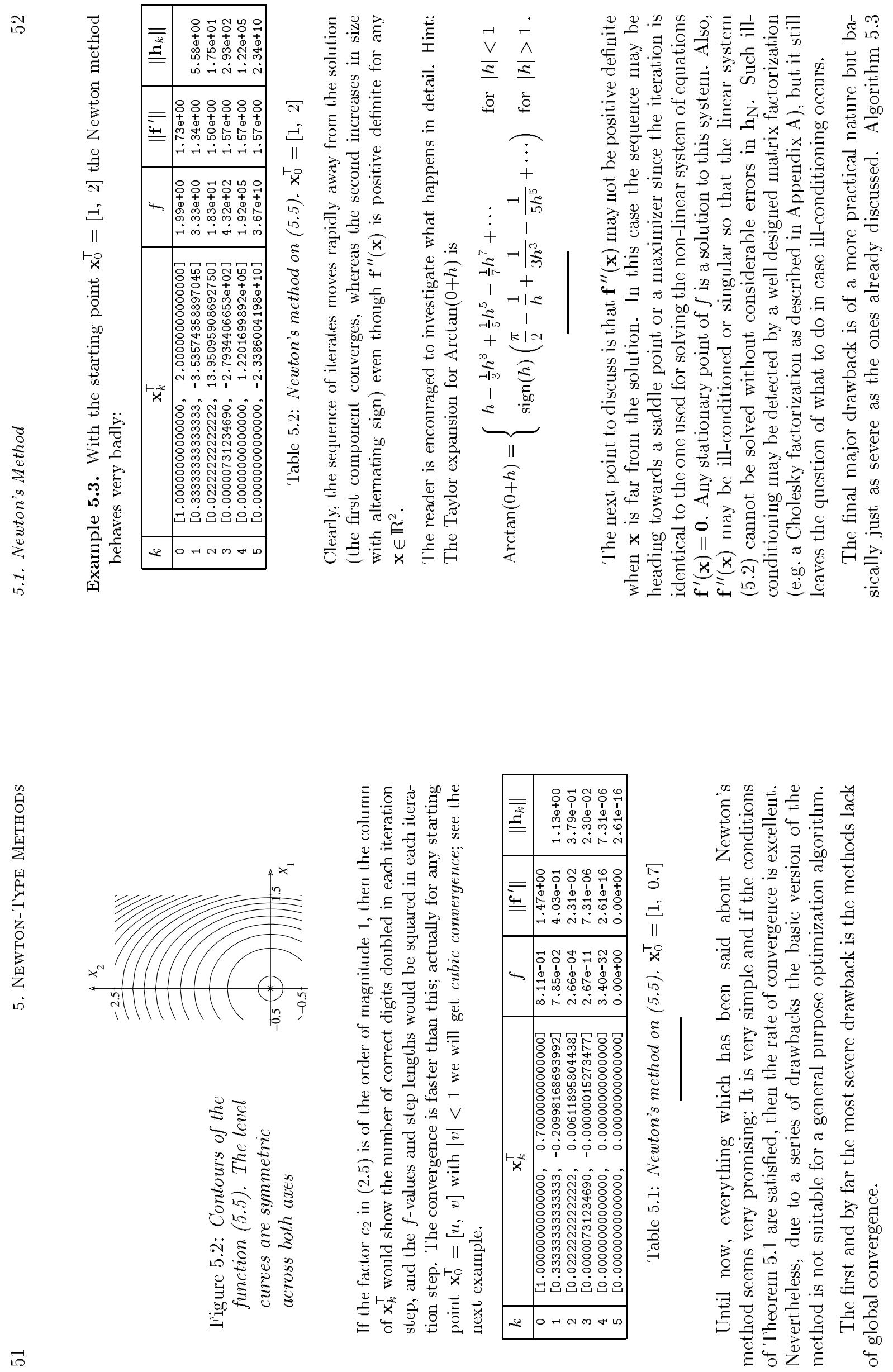

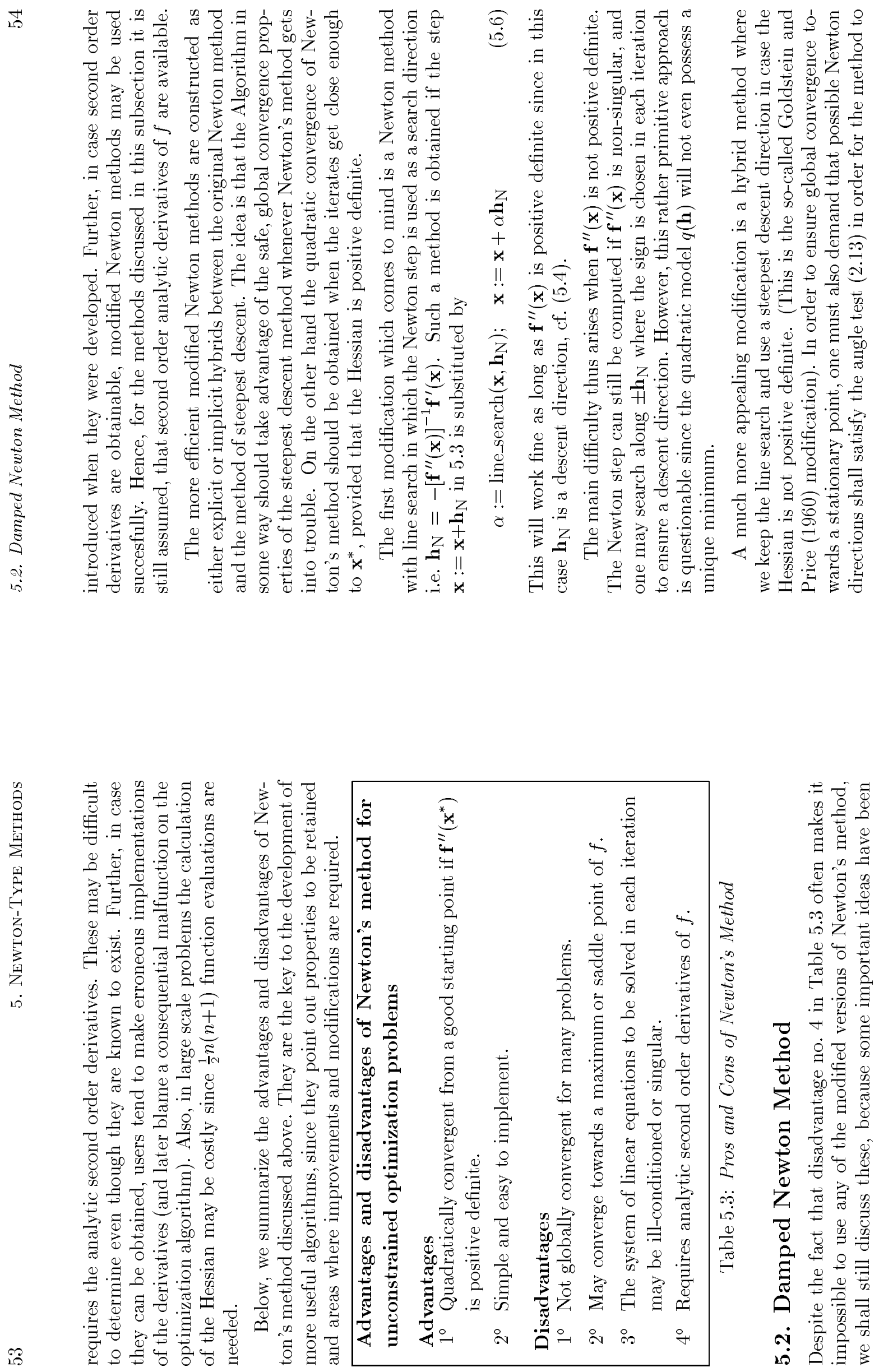

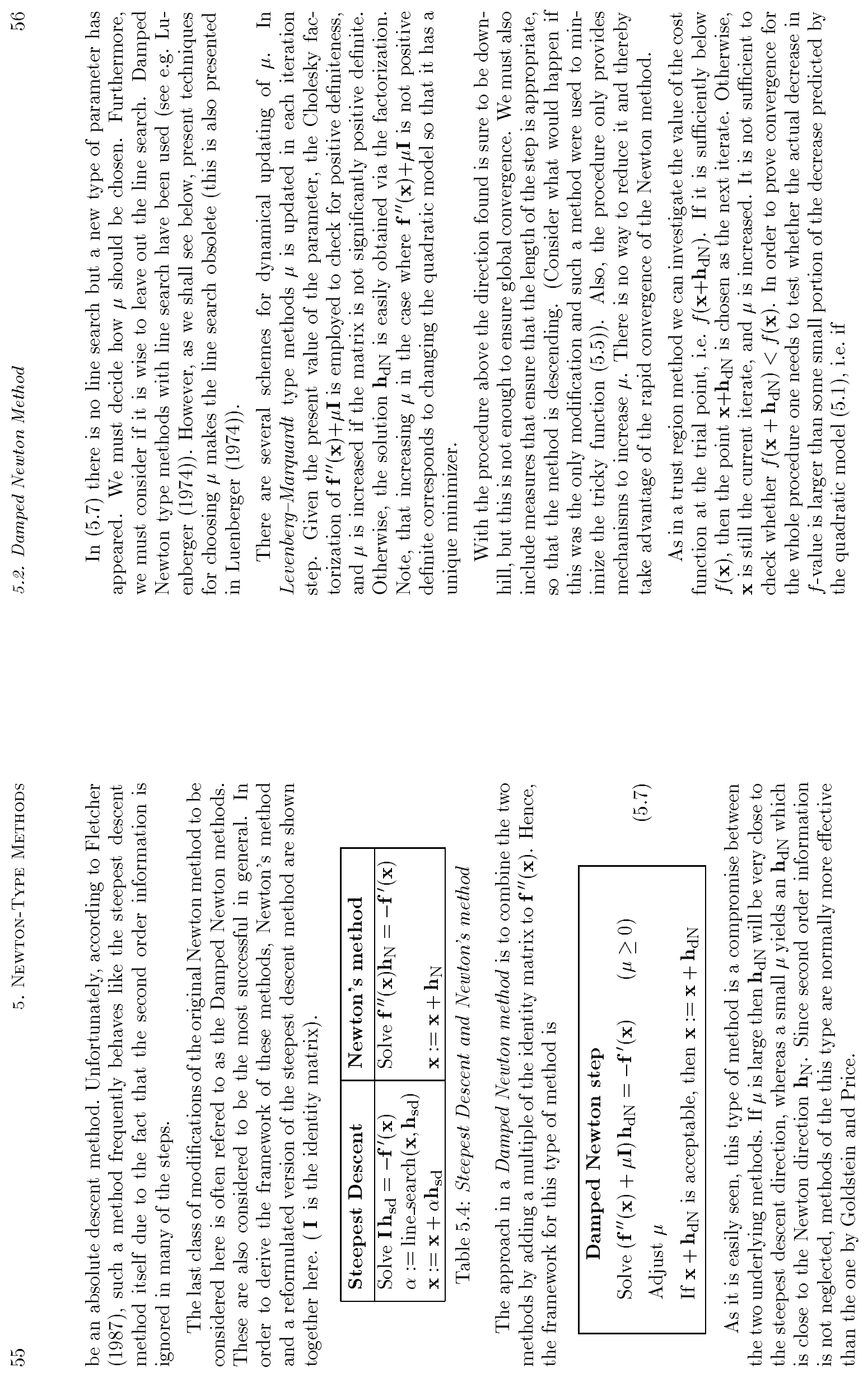


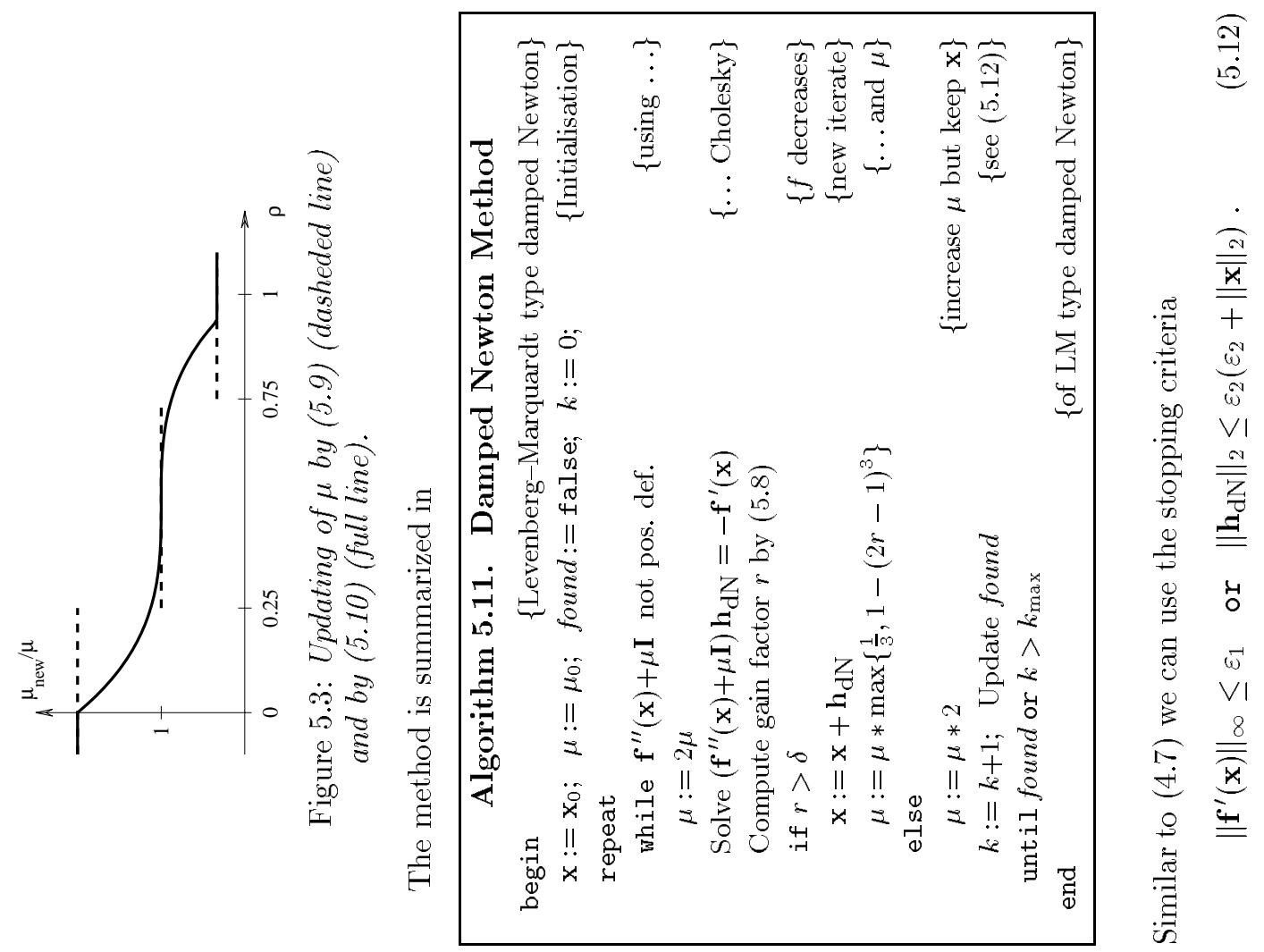

要

15
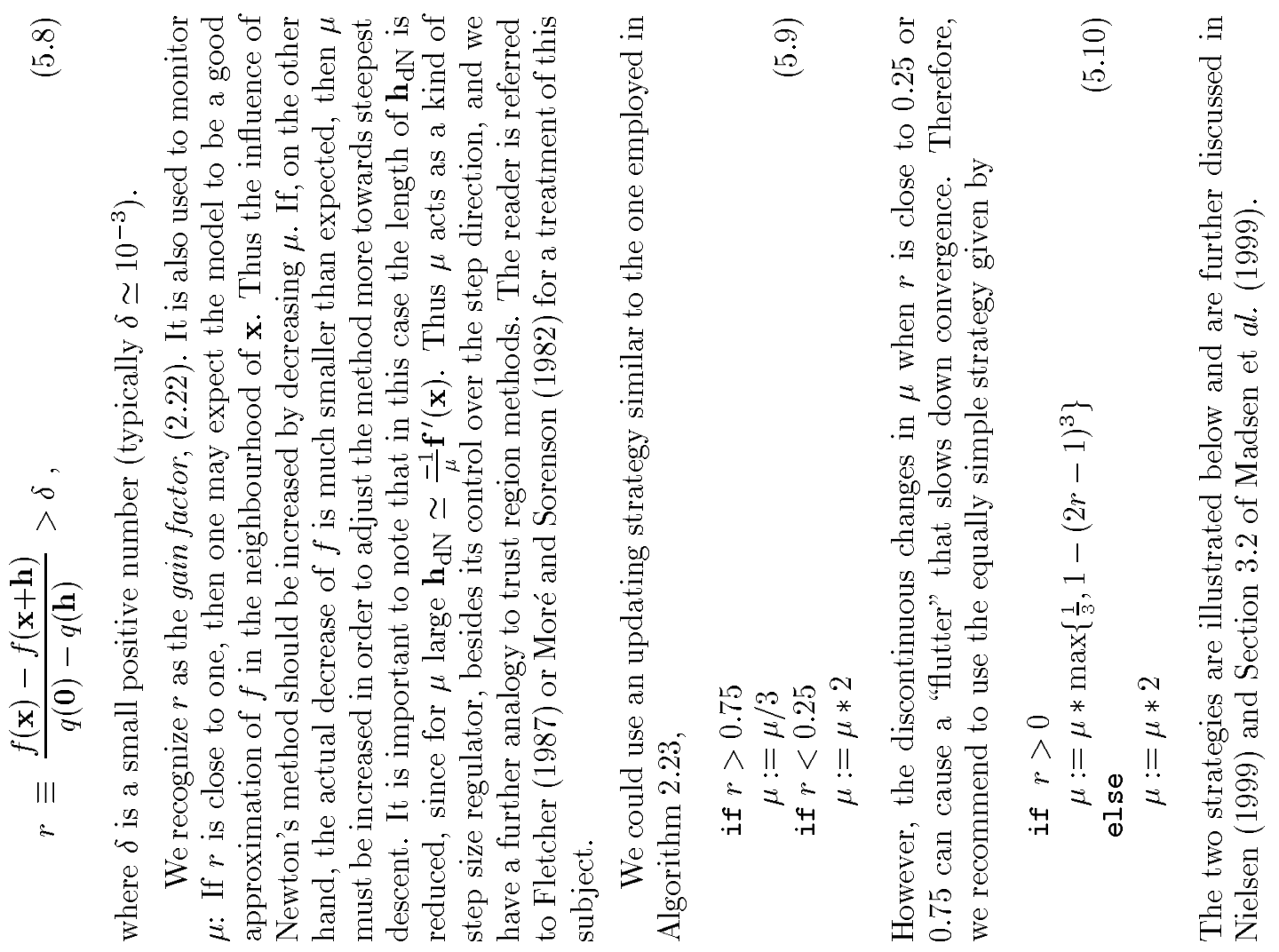
The simplicity of the original Newton method has disappeared in the attempt to obtain global convergence, but this type of method does perform well in general.

Example 5.4. Table 5.5 illustrates the performance of Algorithm 5.11 when applied to the tricky function (5.5). We use $\mu_{0}=1$ and $\varepsilon_{1}=10^{-8}$, $\varepsilon_{2}=10^{-12}$ in (5.12).

\begin{tabular}{|c|c|c|c|c|c|}
\hline$k$ & $\mathbf{x}_{k}^{\top}$ & $f$ & $\left\|\mathbf{f}^{\prime}\right\|_{\infty}$ & $r$ & $\mu$ \\
\hline 0 & {$[1.00000000000,2.00000000000]$} & $1.99 e+00$ & $1.33 \mathrm{e}+00$ & 0.999 & $1.00 e+00$ \\
\hline 1 & {$[0.5555555556,1.0773760685]$} & $6.63 e-01$ & $8.23 e-01$ & 0.872 & $3.33 e-01$ \\
\hline 2 & {$[0.1824004456,0.0441028668]$} & $1.77 e-02$ & $1.84 \mathrm{e}-01$ & 1.010 & $1.96 \mathrm{e}-01$ \\
\hline 3 & {$[0.0323940533,0.0071966616]$} & $5.51 e-04$ & $3.24 e-02$ & 1.000 & $6.54 \mathrm{e}-02$ \\
\hline 4 & {$[0.0020074933,0.0004414865]$} & $2.11 \mathrm{e}-06$ & $2.01 \mathrm{e}-03$ & 1.000 & $2.18 \mathrm{e}-02$ \\
\hline 5 & {$[0.0000428275,0.00000094174]$} & $9.61 \mathrm{e}-10$ & $4.28 \mathrm{e}-05$ & 1.000 & $7.27 e-03$ \\
\hline 6 & {$[0.0000003089,0.0000000679]$} & $5.00 e-14$ & $3.09 \mathrm{e}-07$ & 1.000 & $2.42 e-03$ \\
\hline 7 & {$[0.0000000007,0.0000000002]$} & $3.05 e-19$ & $7.46 e-10$ & & \\
\hline
\end{tabular}

Table 5.5: Algorithm 5.11 applied to (5.5). $\mathbf{x}_{0}^{\top}=[1,2], \mu_{0}=1$

The solution is found without problems, and the columns with $f$ and $\left\|\mathbf{f}^{\prime}\right\|$ show superlinear convergence, as defined in (2.6).

Example 5.5. We have used Algorithm 5.11 on Rosenbrock's function from Example 4.3. We use the same starting point, $\mathrm{x}_{0}=[-1.2,1]^{\top}$, and with $\mu_{0}=1, \varepsilon_{1}=10^{-10}, \varepsilon_{2}=10^{-12}$ we found the solution after 29 iteration steps. The performance is illustrated below

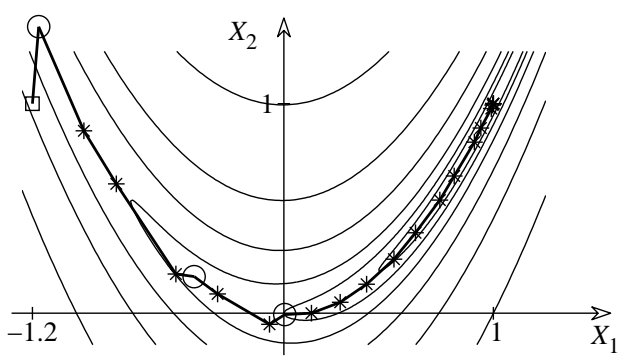

Figure 5.4a: Damped Newton Method on Rosenbrock's function. Iterates

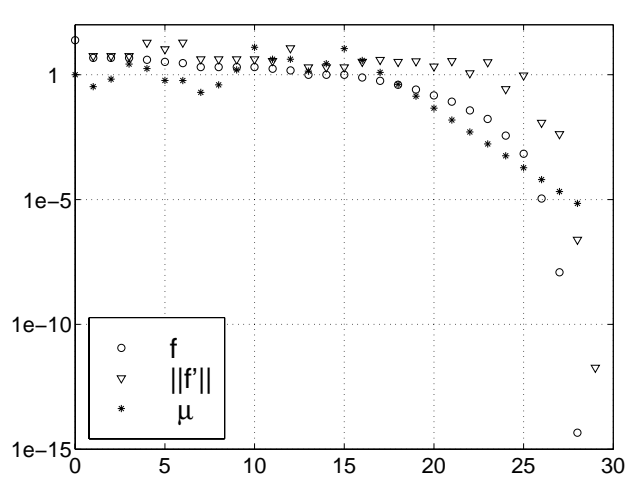

Figure 5.4b: $f\left(\mathbf{x}_{k}\right),\left\|\mathbf{f}^{\prime}\left(\mathbf{x}_{k}\right)\right\|_{\infty}$ and $\mu$.

The three circles in Figure 5.4a indicates points, where the iterations stalls, i.e. the current $\mathrm{x}$ is not changed, but $\mu$ is updated. After passing the bottom of the parabola, the damping parameter $\mu$ is decreased in each step. As in the previous example we achieve superlinear final convergence.

\subsection{Quasi-Newton Methods}

The modifications discussed in the previous section make it possible to overcome the first three of the main disadvantages of Newton's method shown in Table 5.3: The damped Newton method is globally convergent, ill-conditioning may be avoided, and minima are rapidly located. However, no means of overcoming the fourth disadvantage has been considered: The user must still supply formulae and implementations of the second derivatives of the cost function.

In Quasi-Newton methods (from latin, quasi: nearly) the idea is to use matrices which approximate the Hessian matrix or its inverse, instead of the Hessian matrix or its inverse in Newton's equation (5.2). The matrices are normally named

$$
\mathbf{B} \simeq \mathbf{f}^{\prime \prime}(\mathbf{x}) \quad \text { and } \quad \mathbf{D} \simeq \mathbf{f}^{\prime \prime}(\mathbf{x})^{-1}
$$



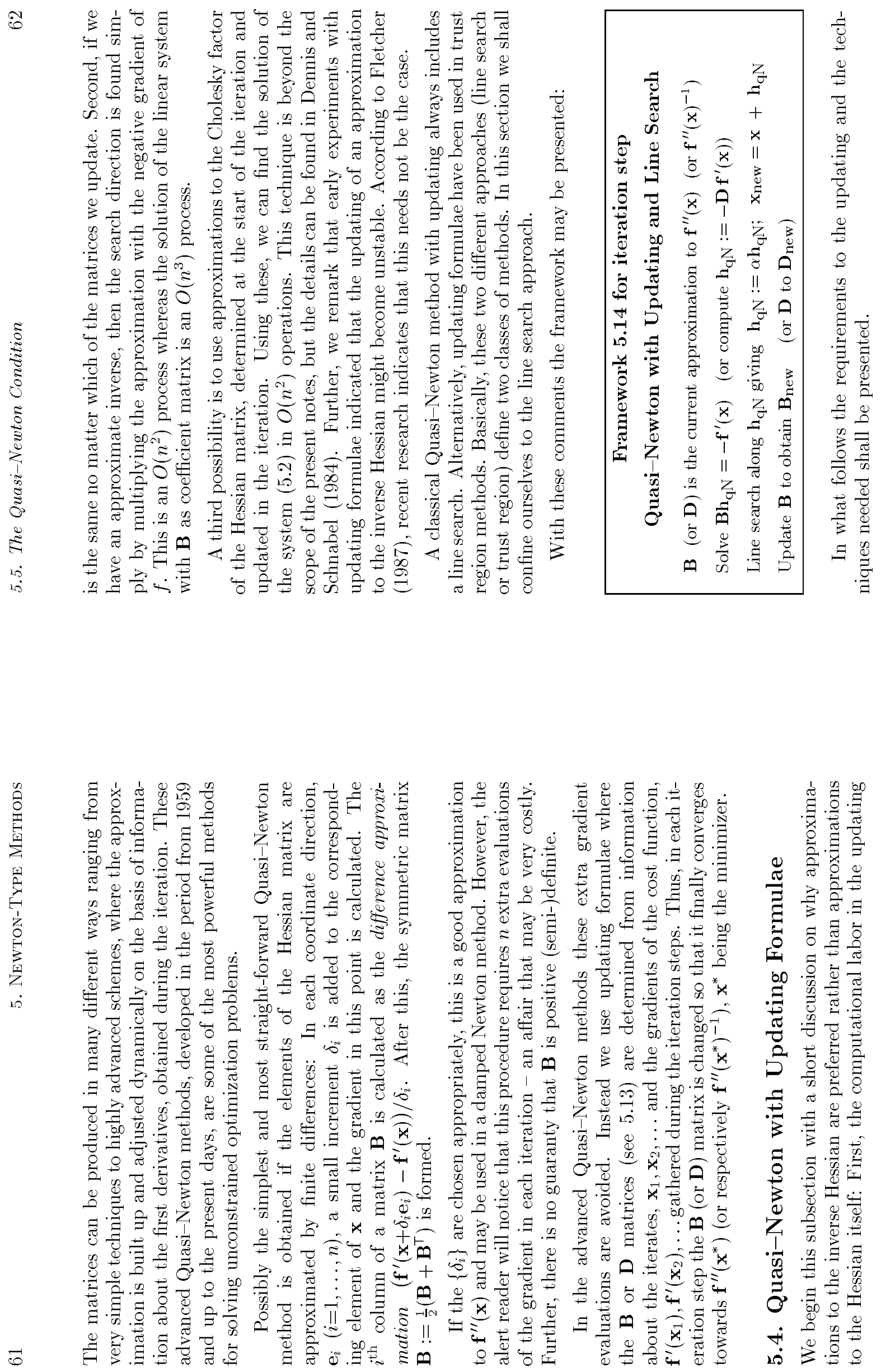

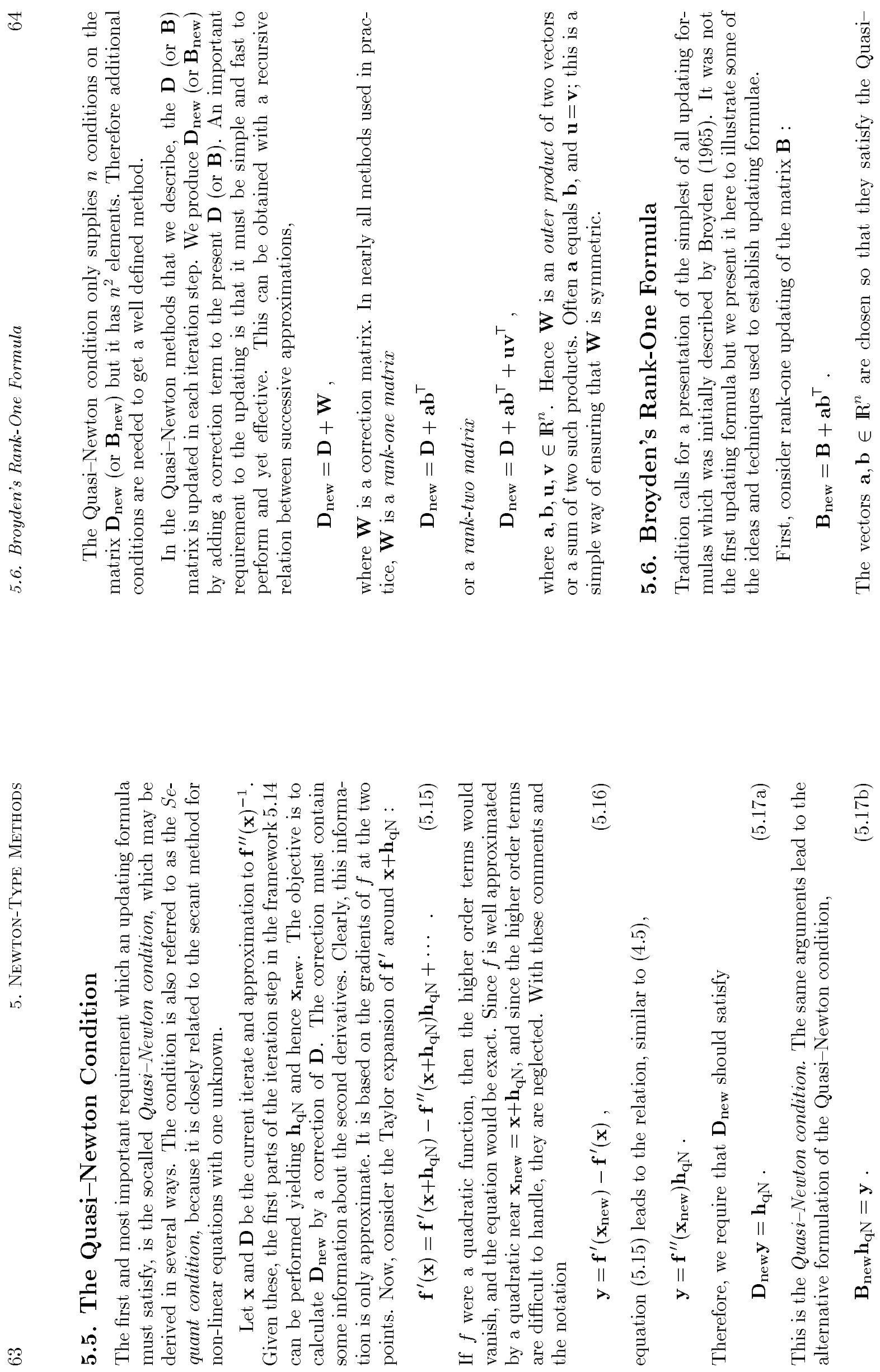


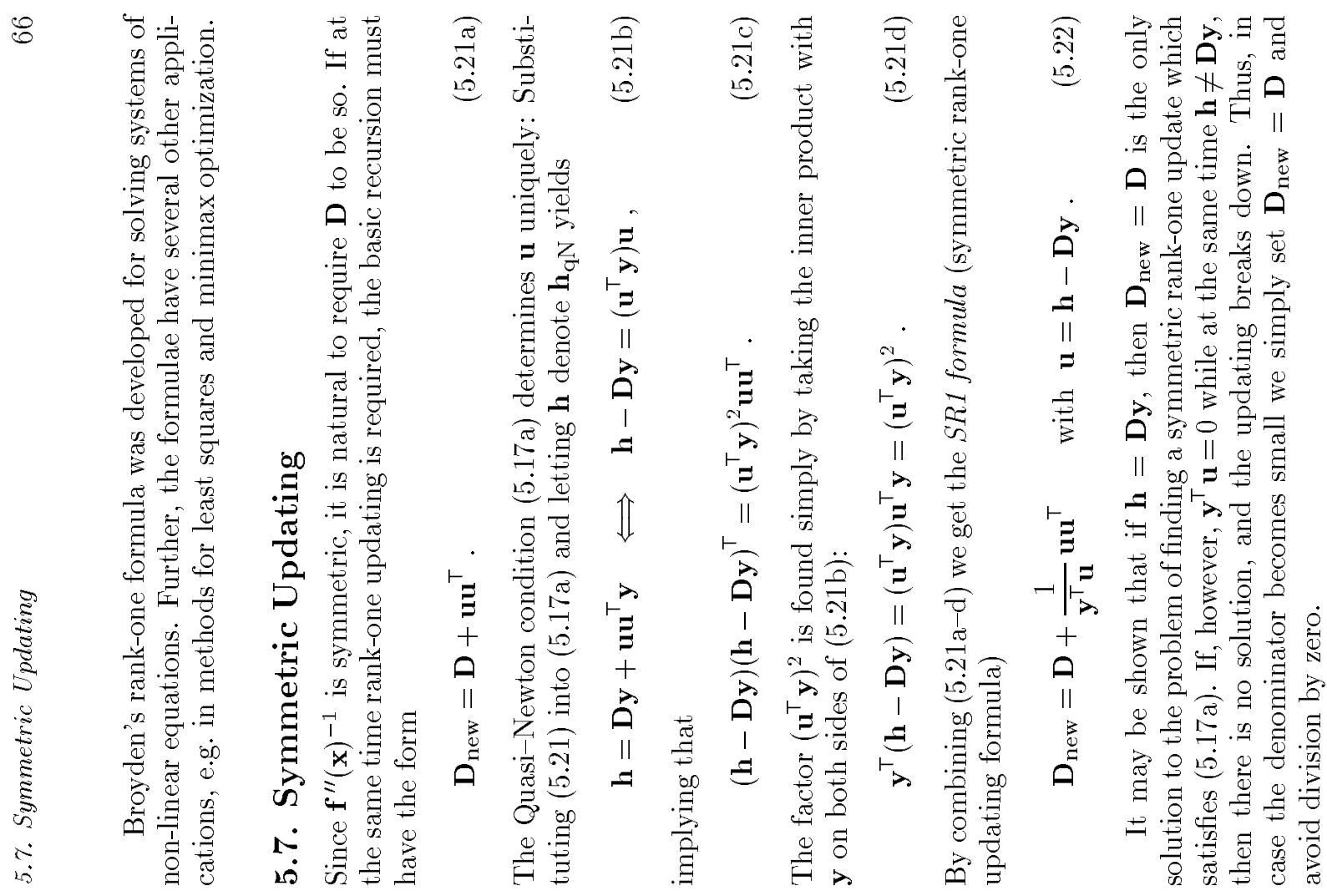

(5)

15

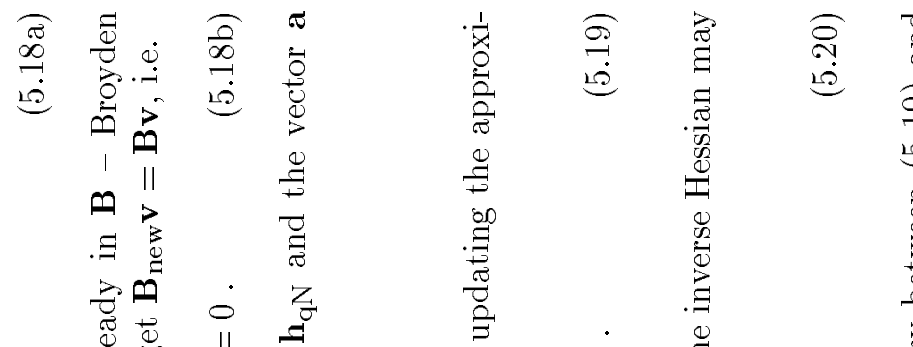

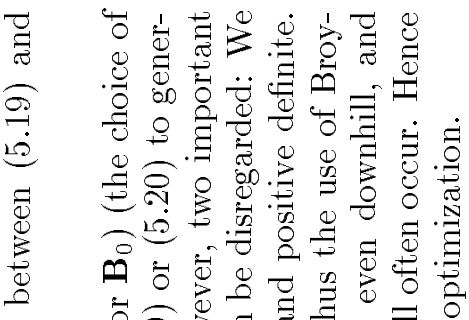

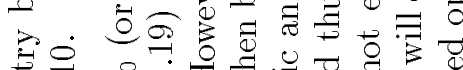

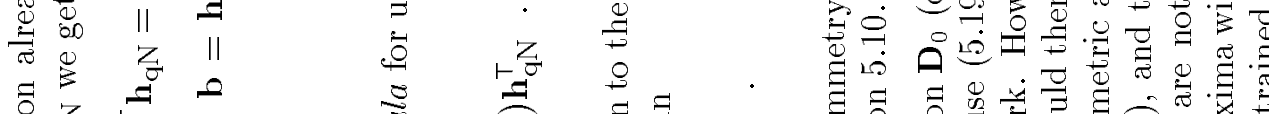

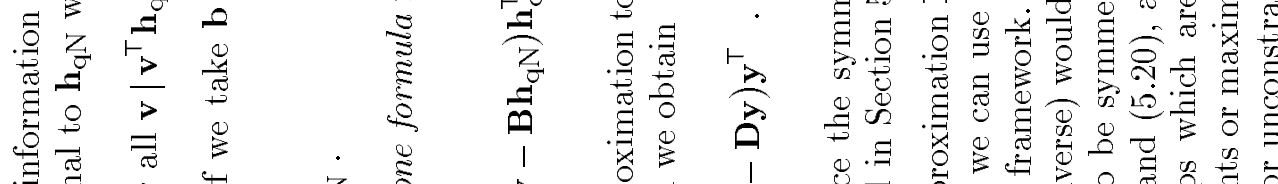

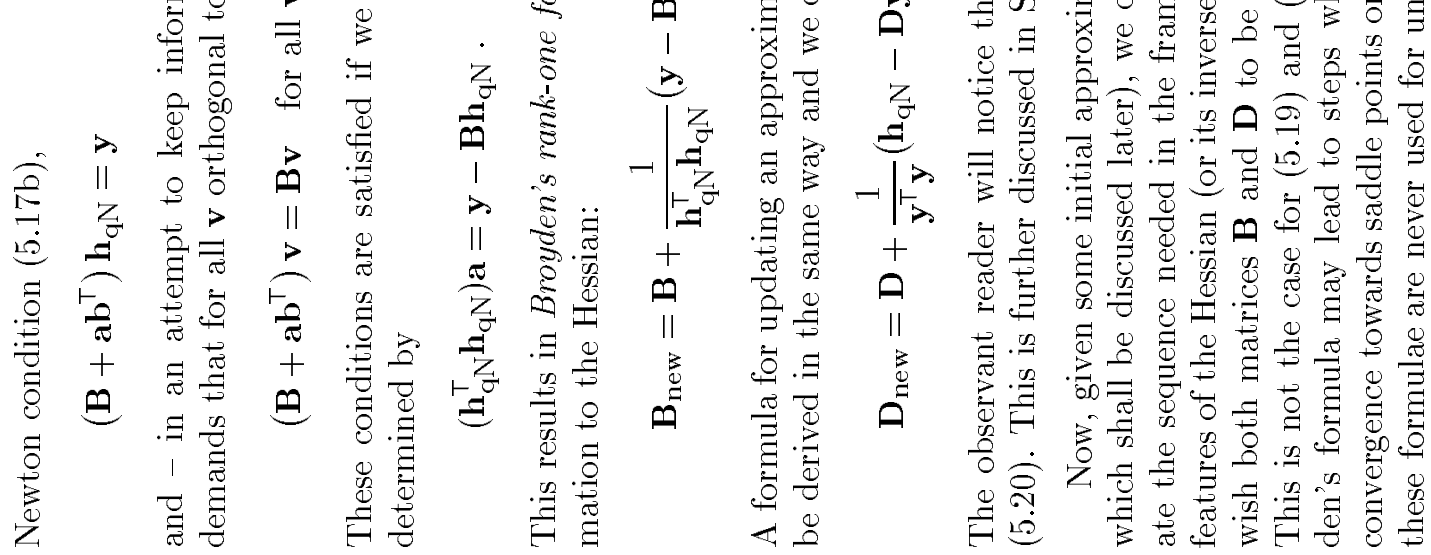



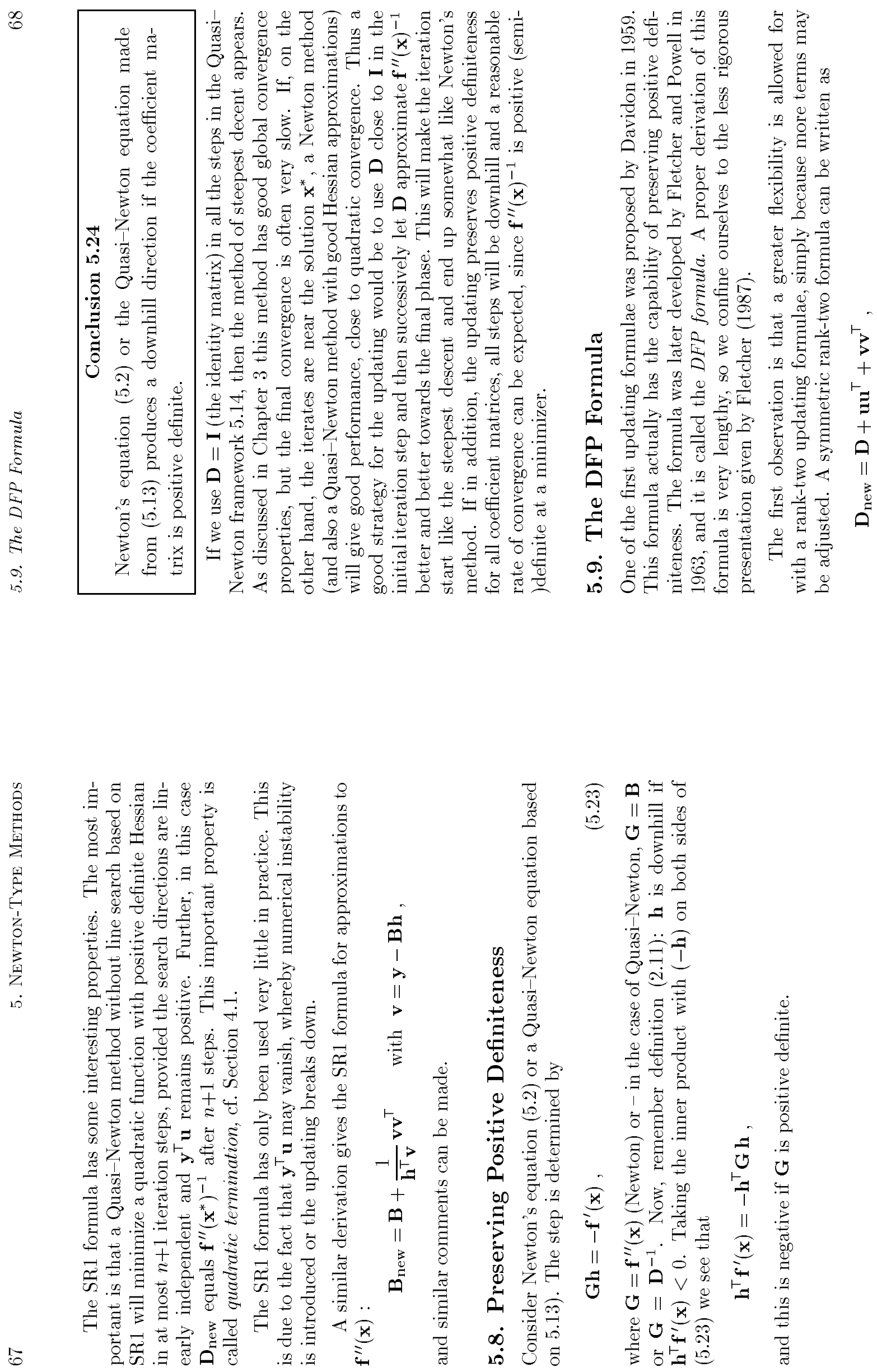

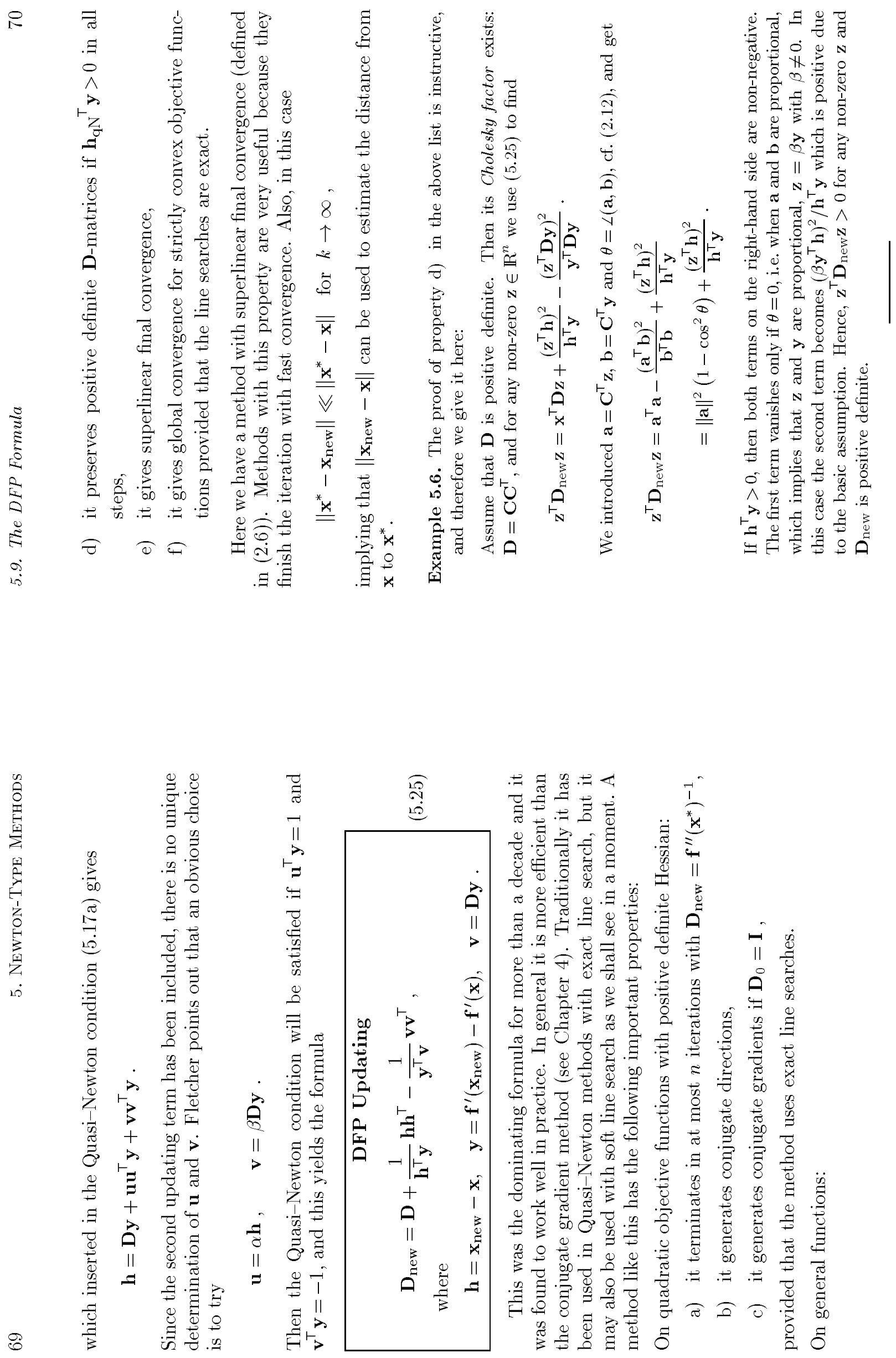

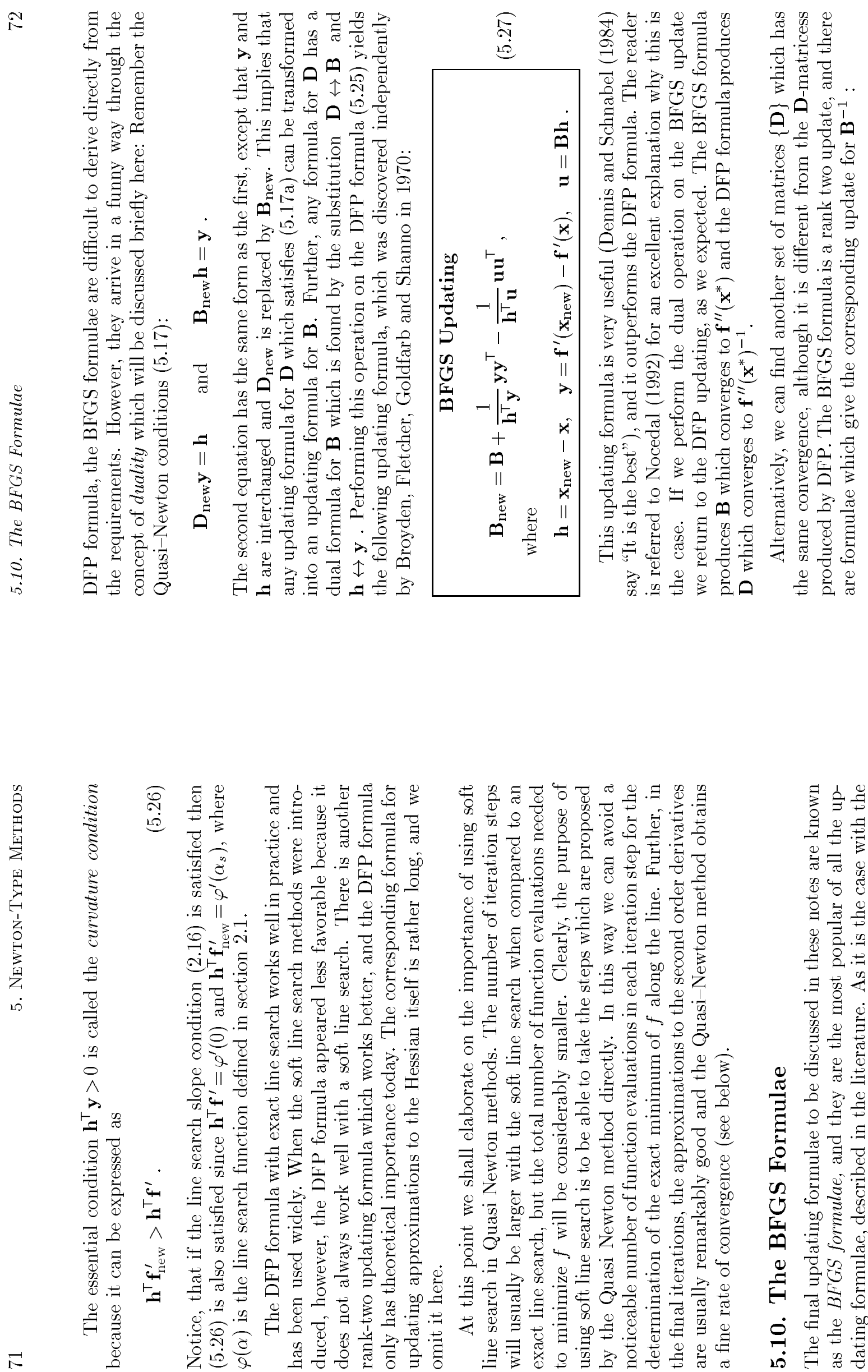

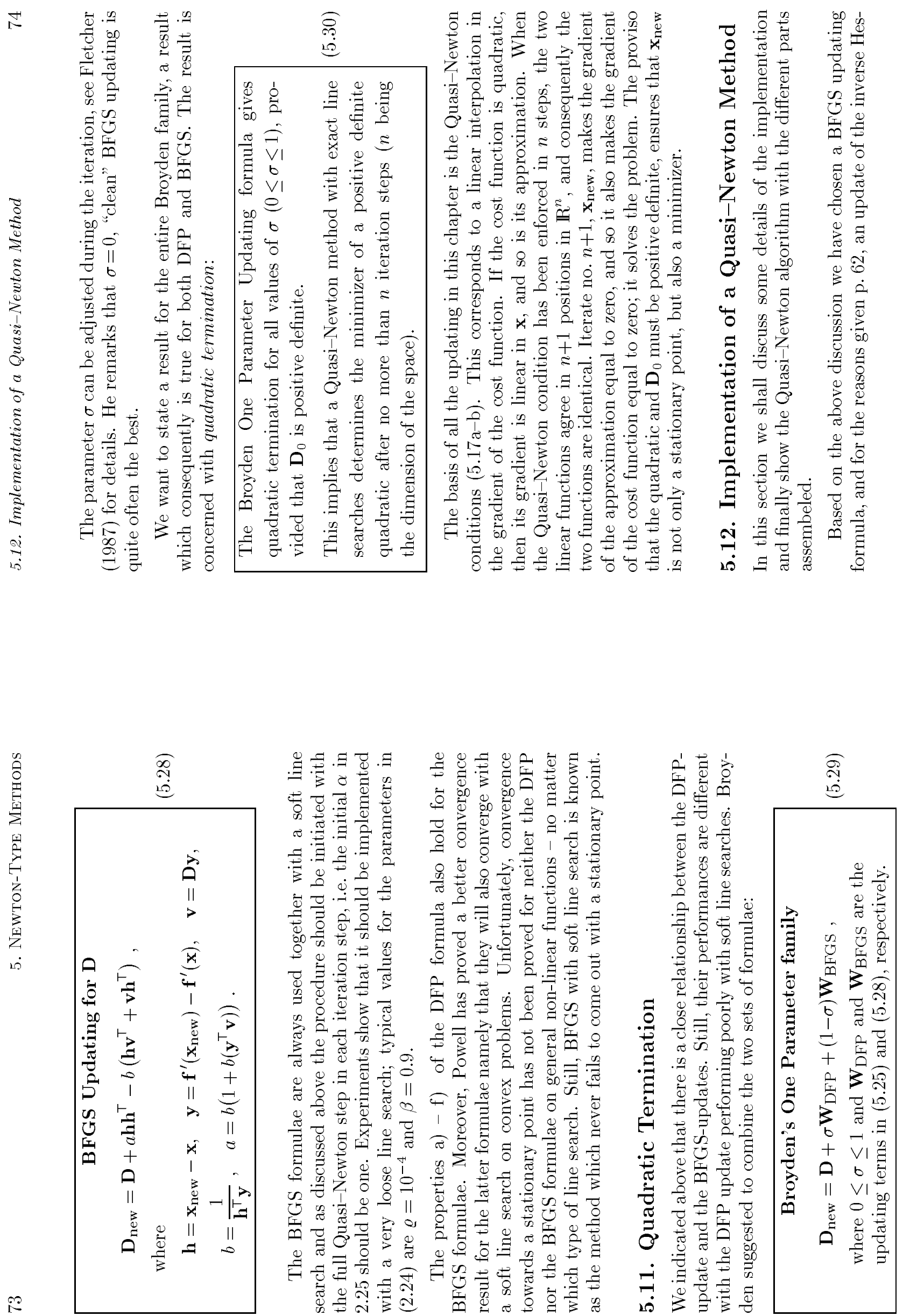

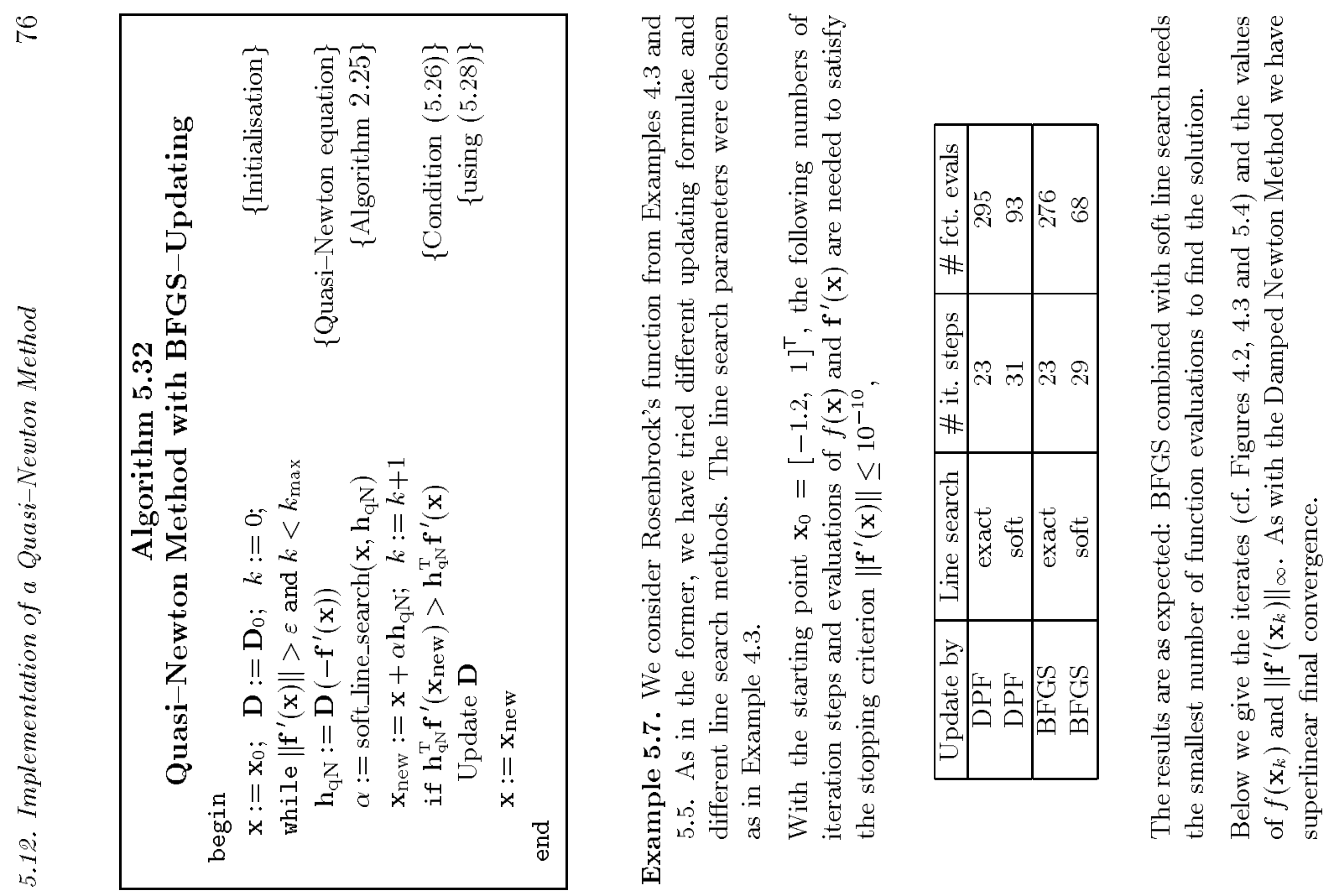

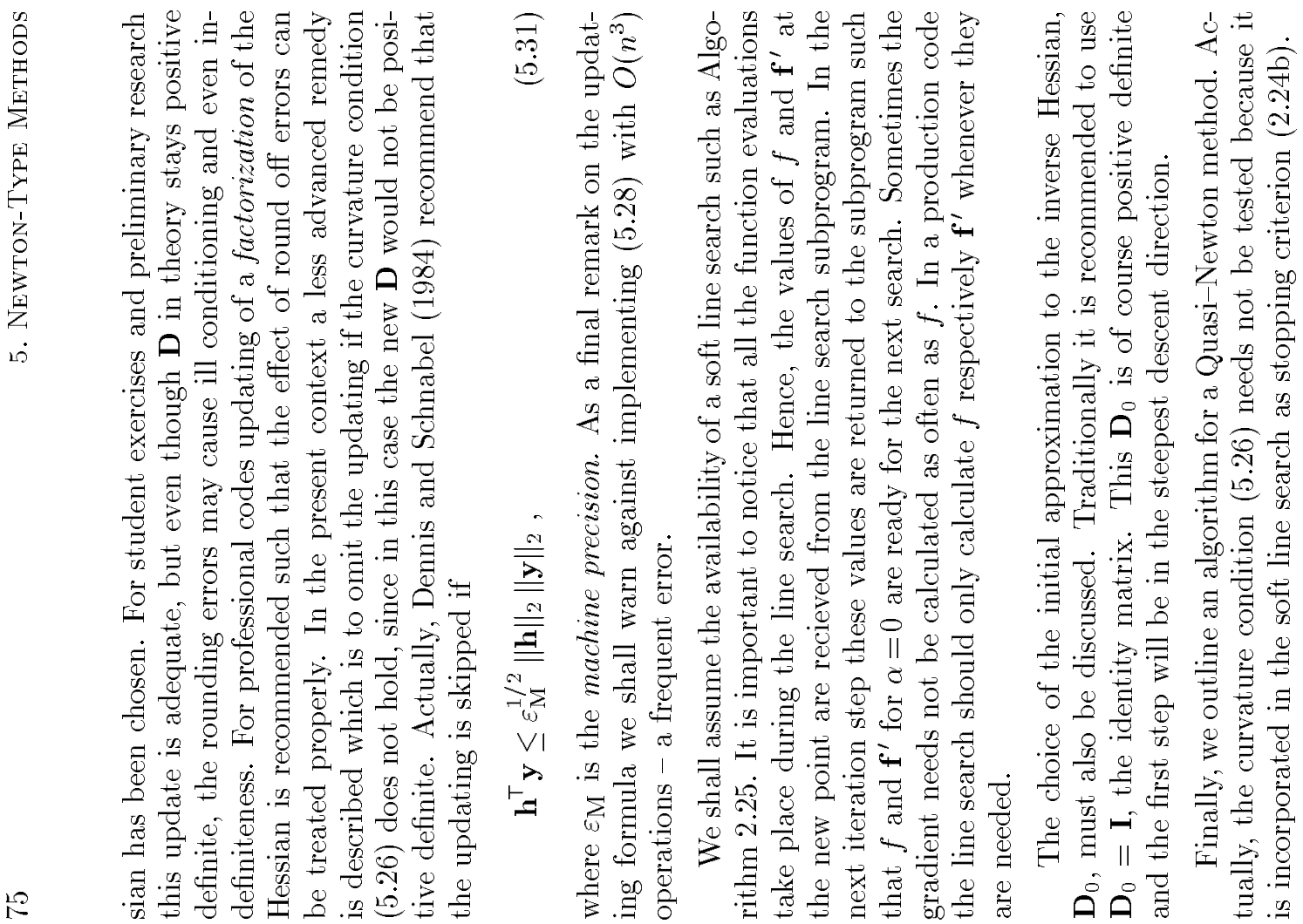



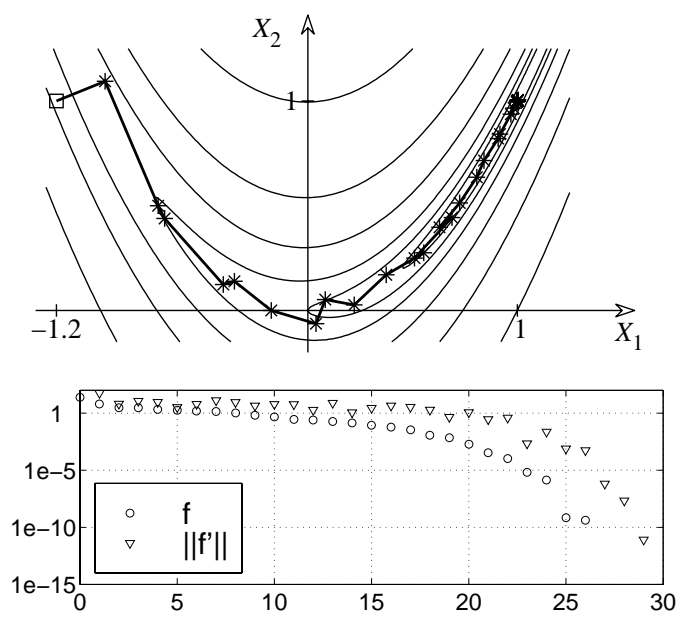

Figure 5.5: BFGS with soft line search

applied to Rosenbrock's function.

Top: iterates $\mathrm{x}_{k} . \quad$ Bottom: $f\left(\mathrm{x}_{k}\right)$ and $\left\|\mathbf{f}^{\prime}\left(\mathrm{x}_{k}\right)\right\|_{\infty}$.

The number of iteration steps is about the same as in Example 5.5, while the number of function evaluations is almost four times as big. Note, however, that with Algorithm 5.32 each evaluation involves $f(\mathrm{x})$ and $\mathrm{f}^{\prime}(\mathrm{x})$, while each evalution in the Damped Newton Method also involves the Hessian $\mathrm{f}^{\prime \prime}(\mathrm{x})$. For many problems this is not available. If it is, it may be costly: we need to compute $\frac{1}{2} n(n+1$ elements in the symmetric matrix $\mathrm{f}^{\prime \prime}(\mathrm{x})$, while $\mathrm{f}^{\prime}(\mathrm{x})$ has $n$ elements only. 

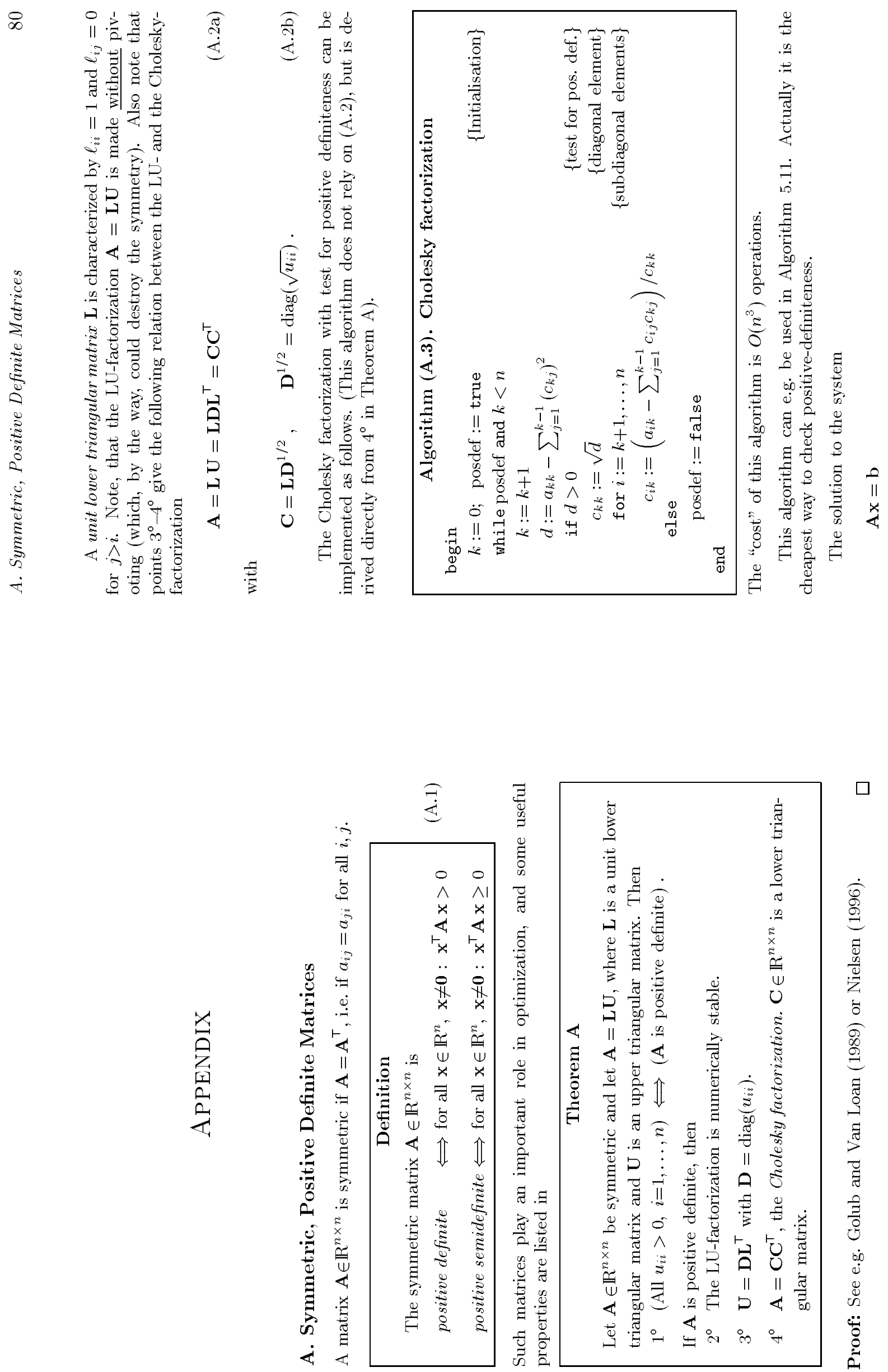

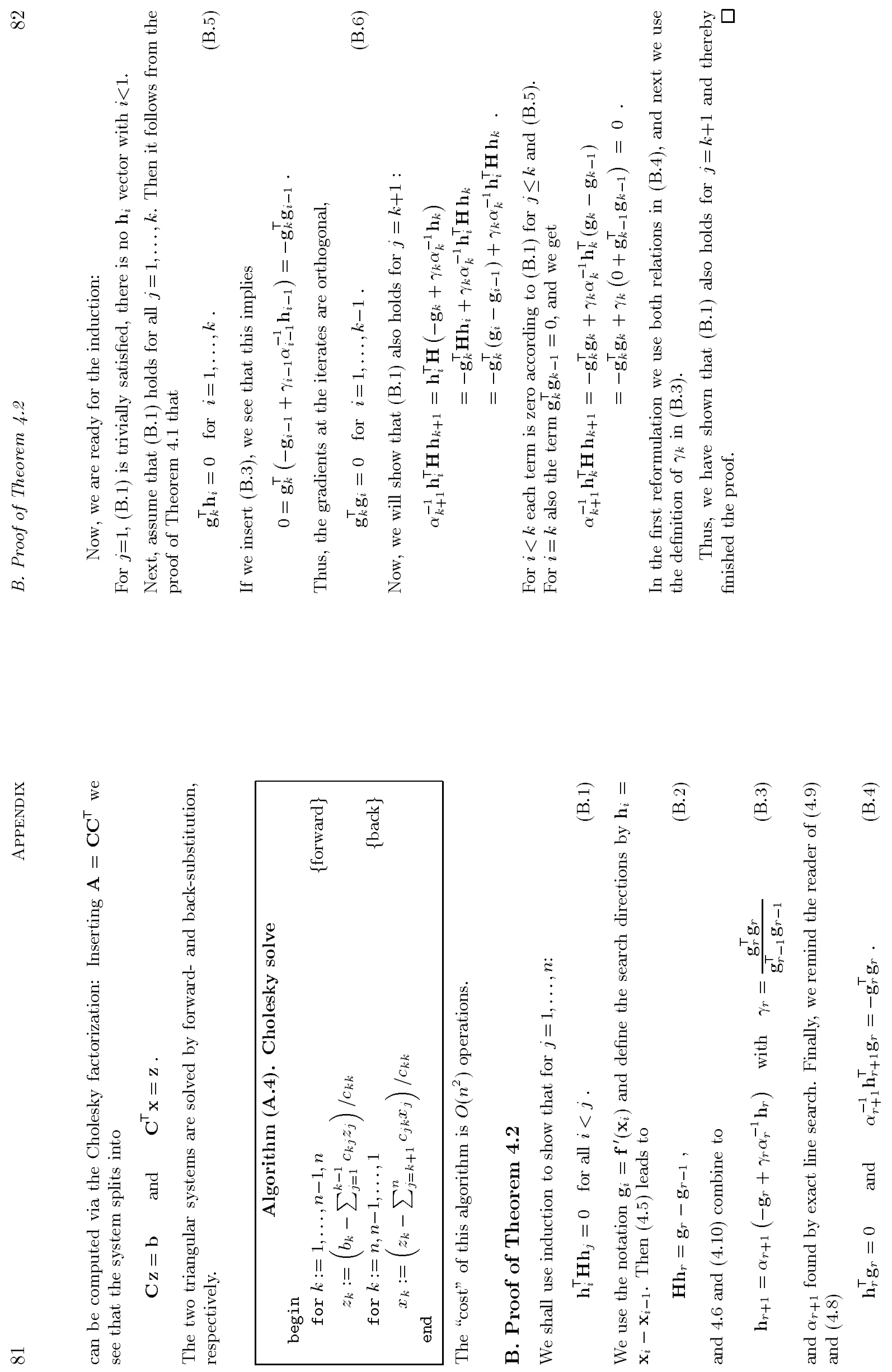

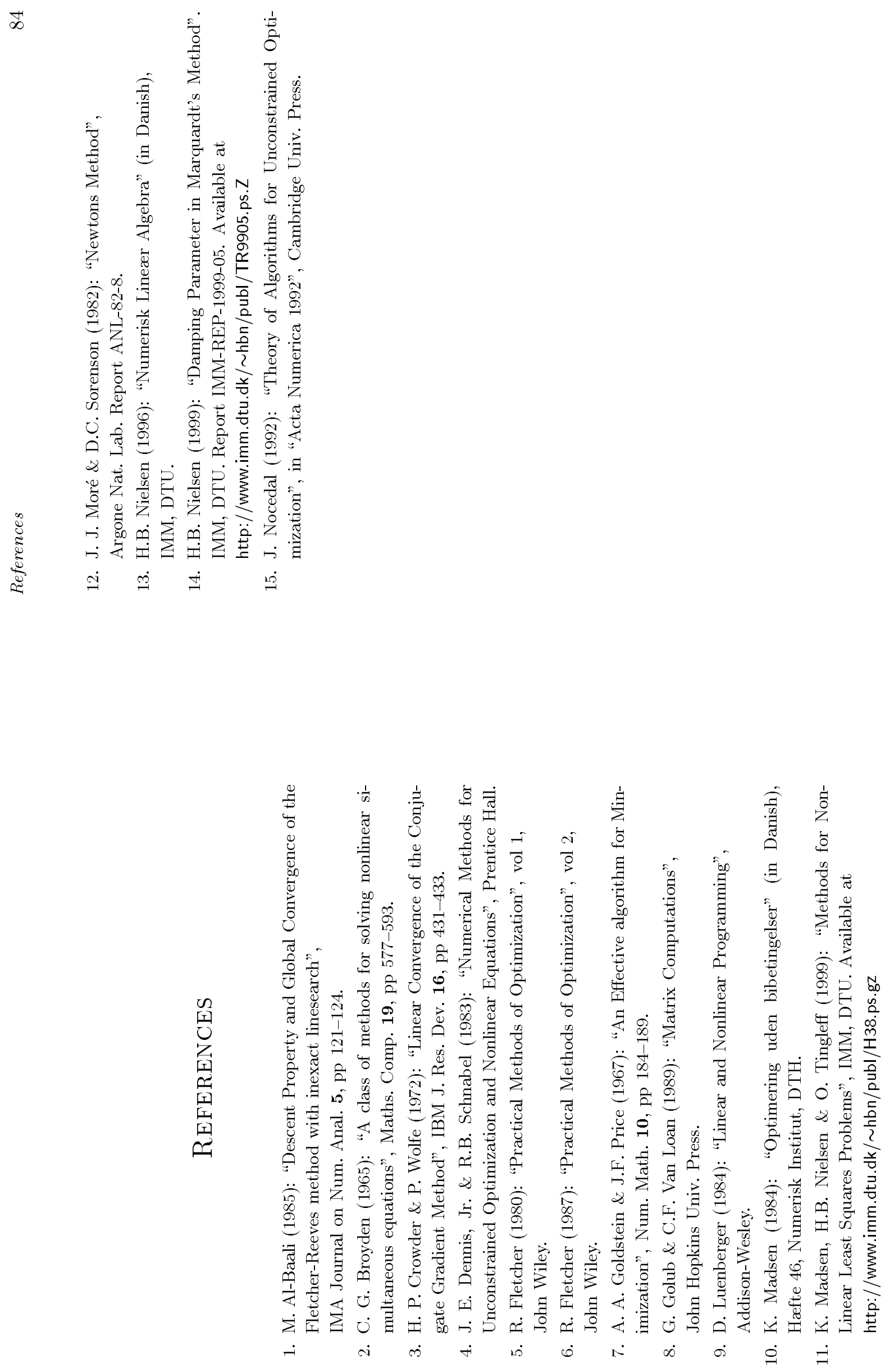


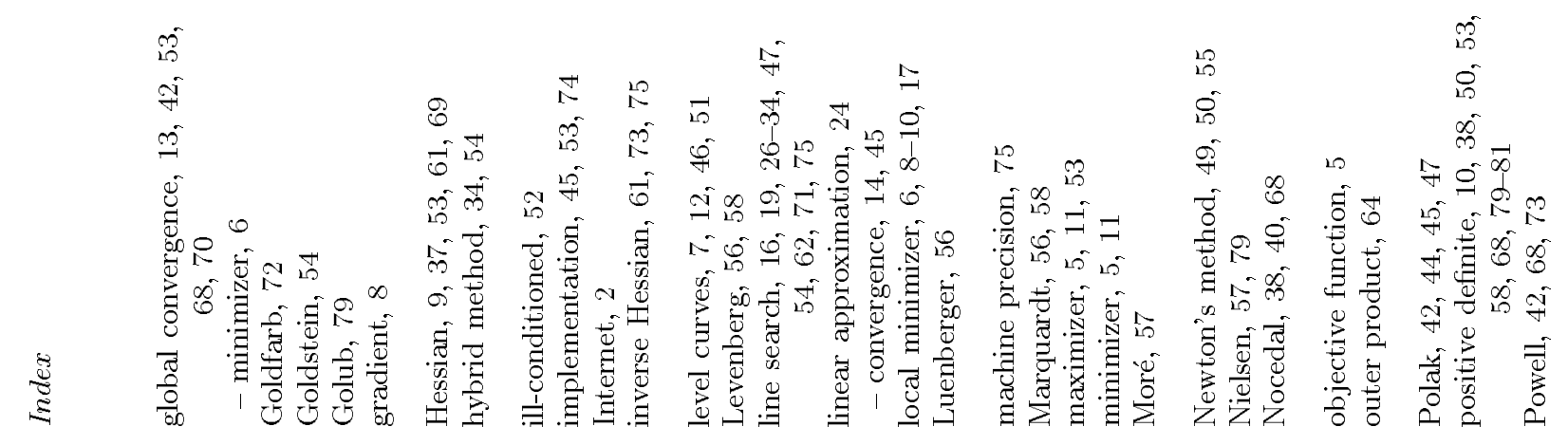

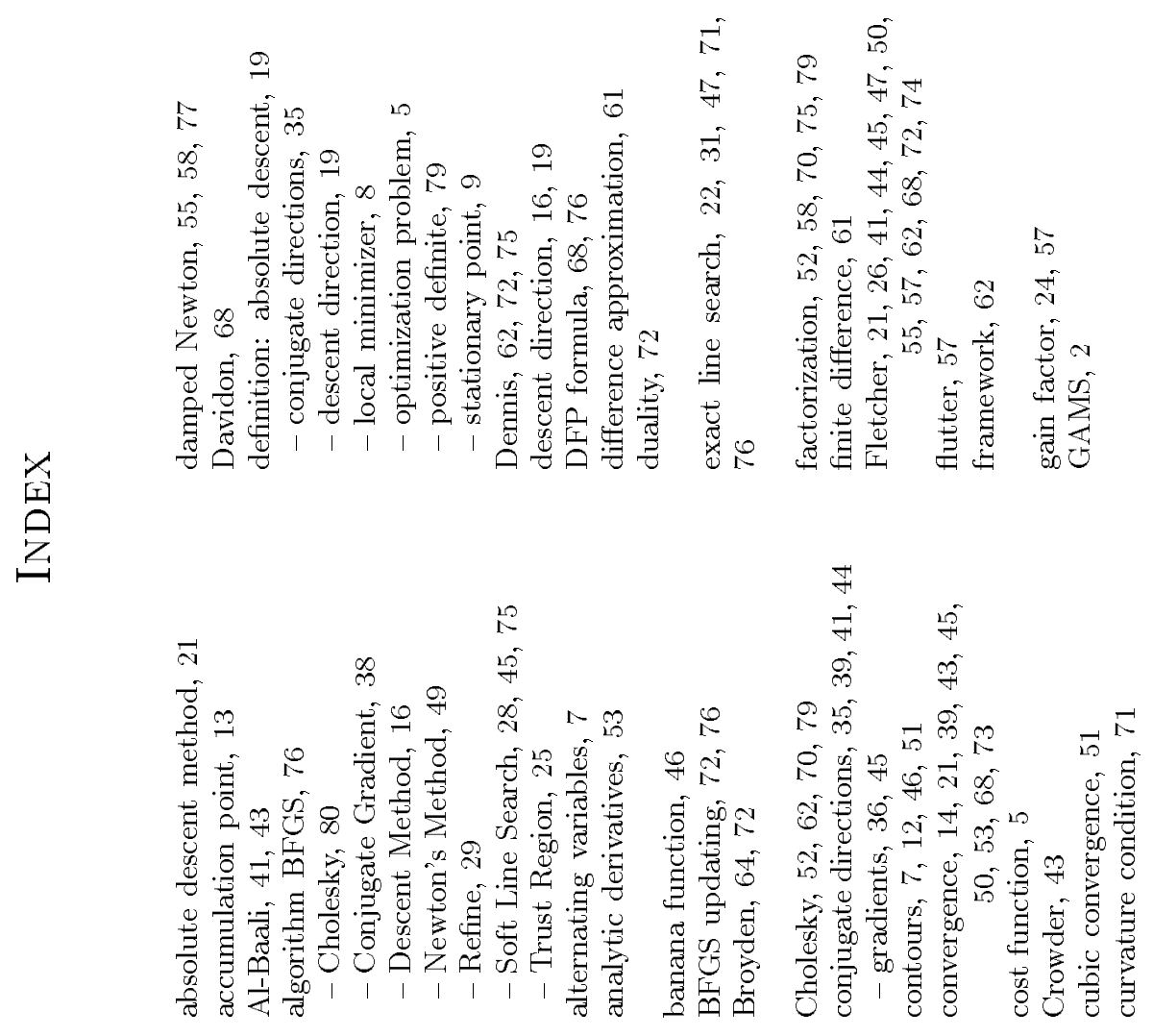

